\title{
REPRESENTATIONS OF ALGEBRAIC GROUPS OVER A 2-DIMENSIONAL LOCAL FIELD
}

\author{
DAVID KAZHDAN AND DENNIS GAITSGORY
}

\begin{abstract}
We introduce a categorical framework for the study of representations of $G(\mathbf{F})$, where $G$ is a reductive group, and $\mathbf{F}$ is a 2-dimensional local field, i.e., $\mathbf{F}=\mathbf{K}((t))$, where $\mathbf{K}$ is a local field.

Our main result says that the space of functions on $G(\mathbf{F})$, which is an object of a suitable category of representations of $G(\mathbf{F})$ with the respect to the action of $G$ on itself by left translations, becomes a representation of a certain central extension of $G(\mathbf{F})$, when we consider the action by right translations.
\end{abstract}

\section{INTRODUCTION}

0.1. Let $\mathbf{K}$ be a local field, and let us consider the field $\mathbf{F}=\mathbf{K}((t))$. In his paper [8], Kapranov studied a certain representation of the group $G(\mathbf{F})$, where $G$ is a reductive group over $\mathbf{K}$. He introduced a pro-vector space (we will denote it by $\mathbb{V}$ ), on which the group $G(\mathbf{F})$ acts in a continuous way, and which may be thought of as an analogue of a principal series representation of usual p-adic groups.

Namely, $\mathbb{V}$ is the (pro)-vector space of locally constant functions with compact support on the set of $\mathbf{K}$-points on the base affine space of the loop group $G((t))$. (We remind that this base affine space is a principal $T$-bundle over the affine flag scheme corresponding to $G$, where $T$ is the Cartan subgroup.)

Kapranov wrote down a certain algebra of endomorphisms of $\mathbb{V}$ generated by explicit intertwining operators, and proved that this algebra is isomorphic to the (modified) double affine Hecke algebra. This double affine Hecke algebra, which was introduced and studied by Cherednik, is clearly an object of great importance, and Kapranov's work explained that it is related to groups over a 2-dimensional field, such as $\mathbf{F}$, in the same way as the usual affine Hecke algebra is related to $\mathfrak{p}$-adic groups.

0.2. The present paper grew out of an attempt to put Kapranov's ideas and results into a categorical framework. Our goal is to find a category of smooth representations, let us denote it $\operatorname{Rep}(\mathbb{G})$, which would contain Kapranov's representation (and its close relatives) as objects. Moreover, we want $\operatorname{Rep}(\mathbb{G})$ to be abelian, so that the usual representation-theoretic questions, such as irreducibility, would make sense in it. We also want $\operatorname{Rep}(\mathbb{G})$ to be as "rigid" or "constrained" as possible, and finally we want the definition of $\operatorname{Rep}(\mathbb{G})$ to resemble the definition of the category of smooth representations for usual p-adic groups.

After some categorical preliminaries in Sect. 1] we propose a definition of Rep(G) in Sect. 2 A somewhat surprising feature of $\operatorname{Rep}(\mathbb{G})$ is that, unlike most abelian categories that arise in representation theory, the natural forgetful functor defined on $\operatorname{Rep}(\mathbb{G})$ does not map to the category of vector spaces, but rather to the category $\mathbb{V e c t}$ of pro-vector spaces. We remark that for the purposes of this paper, one could restrict to the subcategory $\mathbb{V} e c t^{\aleph_{0}}$ of projective systems indexed by countable sets. 
Let us recall that $\mathbb{V} e c t$ is an abelian category, but it is not semi-simple. In fact, the subcategory $\mathbb{V e c t}{ }^{\aleph_{0}}$ has cohomological dimension $\leq 1$, and it can be visualized as follows: An object of $\mathbb{V} e c t^{\aleph_{0}}$ is called strict if it can be represented as a (filtering, countable) inverse system of vector spaces $\mathbf{V}_{i}$, such that the arrows $\mathbf{V}_{i} \rightarrow \mathbf{V}_{j}$ are surjective. Strict objects of $\mathbb{V} e c t^{\aleph_{0}}$ are the same as vector spaces endowed with a linear topology, with a countable fundamental system of neighbourhoods of zero, in which they are separated and complete. However, as is well-known, the category of such topological vector spaces is not abelian, which corresponds to the fact that strict objects of $\mathbb{V e c t} t^{\aleph_{0}}$ do not form an abelian subcategory.

We do have a (left-exact) functor limProj: Vect $\rightarrow$ Vect, but the point of view taken in this paper, and which is largely borrowed from [8, is that we really have to work with the abelian category $\mathbb{V} e c t$, and avoid taking projective limits.

We justify the appearance of $\mathbb{V} e c t$ by showing that $G(\mathbf{F})$ does not have representations in any reasonable sense, unless we admit pro-vector spaces.

0.3. In Sect. 3 we show, generalizing the basic construction of $[8$, how to produce non-trivial objects of $\operatorname{Rep}(\mathbb{G})$.

Namely, let $\mathbb{H}$ be a subgroup of $G(\mathbf{F})$, contained in the group $G[[t]](\mathbf{K})$ of $\mathbf{K}$-points of the group $G[[t]]$ and equal to the preimage of a closed subgroup of $\left(G[[t]] / G^{i}\right)(\mathbf{K})$ for some congruence subgroup $G^{i}$. Then, representations of $\mathbb{H}$ on vector spaces, as well as on pro-vector spaces, are notions that are easy to recover from the usual representation theory of $\mathfrak{p}$-adic groups.

We define two functors $\widetilde{i}_{\mathbb{H}}^{\mathbb{G}}$ and $i_{\mathbb{H}}^{\mathbb{G}}$ from $\operatorname{Rep}(\mathbb{H}, \mathbb{V} e c t)$ to $\operatorname{Rep}(\mathbb{G})$, such that the former is the right adjoint to the tautological restriction functor $\operatorname{Rep}(\mathbb{H}, \mathbb{V e c t}) \rightarrow \operatorname{Rep}(\mathbb{G})$. (In other words, $\widetilde{i}_{\mathbb{H}}^{\mathbb{G}}$ should be thought of as an ordinary induction functor, whereas we think of $i_{\mathbb{H}}^{\mathbb{G}}$ as some sort of semi-infinite induction, by analogy with the theory of modules over vertex algebras, cf. 11.)

Kapranov's representation $\mathbb{V}$ is exactly of the form $i_{\mathbb{H}}^{\mathbb{G}}(\mathbb{C})$, where $\mathbb{H}$ is the group of $\mathbf{K}$-points of the unipotent radical of the Iwahori subgroup of $G((t))$, and $\mathbb{C}$ is the trivial representation. In Sect. 4 we give a slight improvement of Kapranov's main result by showing that the (modified) Cherednik's algebra maps isomorphically onto the ring $\operatorname{End}_{\operatorname{Rep}(\mathbb{G})}(\mathbb{V})$.

In addition, in Sect. [4 we discuss another series of examples of objects of $\operatorname{Rep}(\mathbb{G})$ by applying the functor $i_{\mathbb{H}}^{\mathbb{G}}$ for $\mathbb{H}=G[[t]](\mathbf{K})$ and $G[[t]](\mathbf{K})$-representations, which are restrictions of irreducible cuspidal representations of the $\mathfrak{p}$-adic group $G(\mathbf{K})$. By analogy with the corresponding result in the theory of $\mathfrak{p}$-adic groups, we conjecture that these objects are actually irreducible in $\operatorname{Rep}(\mathbb{G})$, and give some evidence in support of this conjecture.

0.4. Finally, in Sect. 5 we formulate and prove the main result of this paper.

Suppose that the group $G$ acts on an algebraic variety $S$. In the theory of p-adic groups one introduces the Schwartz space Funct ${ }_{c}^{l c}(S(\mathbf{K}))$ of locally constant compactly supported functions on the set of $\mathbf{K}$-points of $S$, which is a smooth representation of the group $G(\mathbf{K})$.

The question that we want to address is whether one can define an analogue of the Schwartz space, denoted in this paper by $M(\mathbb{S})$, which would be related to functions and/or distributions on the set of $\mathbf{F}$-valued points of $S$. Of course, one expects that $M(\mathbb{S})$ is an object of $\mathbb{V e c t}$, underlying a $G(\mathbf{F})$-representation.

It appears that the answer to this question is negative in the simplest example of $G=$ $S L_{2}$ acting on the projective line, and the situation seems to be analogous to the problem of developing the theory of D-modules on loop spaces, cf. [1].

However, there are two important examples of $G$-varieties $S$, for which we can define $M(\mathbb{S})$ : 
First, we consider the case of $S$ being the affine space $A^{n}$, with the natural action of $G L_{n}$. We introduce a space $M\left(\mathbb{A}^{n}\right)$ and show that it is naturally an object in the category of representations of the group $\widehat{\mathbb{G L}}_{n}$ (here $\widehat{\mathbb{G L}}_{n}$ is the group of $\mathbf{K}$-points of the canonical (i.e., Tate) central extension $\left.1 \rightarrow G_{m} \rightarrow \widehat{G L}_{n} \rightarrow G L_{n}((t)) \rightarrow 1\right)$.

Next, we consider the case when the variety $S$ is isomorphic to the group $G$ itself, with the action by left translations, and we construct an object $M(\mathbb{G}) \in \operatorname{Rep}(\mathbb{G})$. Now the natural question to ask is, whether the action of $G(\mathbf{F})$ on itself by right translations defines on $M(\mathbb{G})$ another, commuting, structure of an object of $\operatorname{Rep}(\mathbb{G})$.

The answer to this question is that the right action of $G(\mathbf{F})$ on $M(\mathbb{G})$ develops an anomaly (compare it with the main theorem from $[1]$ ). Namely, $M(\mathbb{G})$ does carry a commuting action, but of the group of $\mathbf{K}$-points of the central extension $1 \rightarrow G_{m} \rightarrow \widehat{G} \rightarrow G((t)) \rightarrow 1$ corresponding to the adjoint action of $G$ on its Lie algebra.

0.5. Acknowledgments. We would like to thank S. Arkhipov, I. Cherednik, P. Etingof, V.Ginzburg, M. Kapranov for useful discussions and communications. We are grateful to the anonymous reviewer for valuable comments on the previous version of the paper, and to E. Hrushovski for reading the revised version.

The research of D.G. is supported by the long-term fellowship at the Clay Mathematics Institute. He also wants to thank the Mathematics Department of the Hebrew University of Jerusalem, where the main part of this work was written.

\section{Preliminaries}

1.1. We will work with inductive and projective limits of objects of various categories. Let $I$ be a set. Recall that $I$ is said to be filtering if it is endowed with a partial order, such that for any two elements $i_{1}, i_{2} \in I$, there exists an element $i^{\prime} \in I$ with $i^{\prime} \geq i_{1}, i_{2}$.

Let $I$ be a filtering set, which we can regard as a category, and $\Phi: i \mapsto S_{i}$ be a functor $I \rightarrow$ Set. We will denote by $\lim S_{i}$ the inductive limit of $\Phi$. In other words,

$$
\operatorname{Hom}_{S e t}\left(\lim _{\longrightarrow} S_{i}, S\right) \simeq \operatorname{Hom}_{\text {Functors }}\left(\Phi, \Phi_{S}\right),
$$

where Functors denotes the category of functors $I \rightarrow S e t$, and $\Phi_{S}$ is the "constant" functor corresponding to the set $S$.

Let $\mathcal{C}$ be an arbitrary category. Recall from [7] that the ind-completion of $\mathcal{C}$, denoted $\operatorname{Ind}(\mathcal{C})$, is the full subcategory in the category of contravariant functors $\mathcal{C} \rightarrow$ Set, which consists of objects (isomorphic to ones) of the form

$$
X \mapsto \underset{\lim }{\longrightarrow} \operatorname{Hom}_{\mathcal{C}}\left(X, X_{i}\right),
$$

where $i \mapsto X_{i}$ is a functor $I \rightarrow \mathcal{C}$ and $I$ is a filtering set; we will denote by "lim" $X_{i}$ the corresponding object of $\operatorname{Ind}(\mathcal{C})$, which we will call "the direct limit of the system $X_{i}$ ". By definition, "lim" $X_{i}(X)=\stackrel{\lim }{\longrightarrow} \operatorname{Hom}\left(X, X_{i}\right)$, where the inductive limit is taken in the category of sets.

For example, let $V e c t$ (resp., Vect $t_{0}$ ) be the category of vector spaces (resp., finite-dimensional vector spaces) over a given ground field. We have $V e c t \simeq \operatorname{Ind}\left(V e c t_{0}\right)$. (It is a good exercise to show $\operatorname{Ind}(V e c t)$ is NOT equivalent to $V e c t$.)

For a cardinal $\aleph$, we will denote by $\operatorname{Ind}^{\aleph}(\mathcal{C})$ the full subcategory of $\operatorname{Ind}(\mathcal{C})$ obtained by imposing the condition that the sets of indices that we are considering are of cardinality $\leq \aleph$.

We have a canonical fully faithful embedding $\mathcal{C} \rightarrow \operatorname{Ind}(\mathcal{C})$. The (partially defined) left adjoint $\operatorname{Ind}(\mathcal{C}) \rightarrow \mathcal{C}$, called the inductive limit, which we will denote by limInd, is always right-exact. 
We will say that $\mathcal{C}$ is closed under inductive limits (resp., inductive limits of cardinality $\leq \aleph$ ) if the functor $\lim I n d$ is defined on the entire $\operatorname{Ind}(\mathcal{C})\left(\right.$ resp., $\operatorname{Ind}^{\aleph}(\mathcal{C})$ ). For example, it is easy to show that any category of the form $\operatorname{Ind}(\mathcal{C}),\left(\operatorname{resp} ., \operatorname{Ind}^{\aleph}(\mathcal{C})\right)$, where $\mathcal{C}$ is another category, is always closed under inductive limits (resp., of cardinality $\leq \aleph$ ).

Note on the terminology: Let us emphasize that for a functor $I \rightarrow \mathcal{C}: i \mapsto X_{i}$, we denote by "lim" $X_{i}$ the corresponding object of $\operatorname{Ind}(\mathcal{C})$, and call it the direct limit of the $X_{i}$ 's, following [7. By contrast, if the object $\lim \operatorname{Ind}\left(" \lim " X_{i}\right) \in \mathcal{C}$ exists, we will call it the inductive limit of the $X_{i}$ 's, and denote it also by $\underset{\lim }{\longrightarrow} X_{i}$.

The following simple assertion is useful:

Lemma 1.2. Assume that $\mathcal{C}$ is closed under inductive limits of cardinality $\leq \aleph$, and $X \in$ $\operatorname{Ind}^{\aleph}(\mathcal{C})$. Then $X$ belongs to $\mathcal{C}$ if and only if for every "lim" $X_{i}^{\prime}=: X^{\prime} \in \operatorname{Ind}^{\aleph}(\mathcal{C})$, the canonical arrow $X\left(\underset{\lim }{\longrightarrow} X_{i}^{\prime}\right) \rightarrow \underset{\longleftarrow}{\lim }\left(X\left(X_{i}^{\prime}\right)\right) \simeq \operatorname{Hom}_{\operatorname{Ind}(\mathcal{C})}\left(X^{\prime}, X\right) \overrightarrow{\text { is }}$ an isomorphism.

The pro-completion $\operatorname{Pro}(\mathcal{C})$ (resp., $\operatorname{Pro}^{\aleph}(\mathcal{C})$ ) and the functor limProj: $\operatorname{Pro}(\mathcal{C}) \rightarrow \mathcal{C}$ are defined in the same way by inverting the arrows, i.e., $\operatorname{Pro}(\mathcal{C})=\left(\operatorname{Ind}\left(\mathcal{C}^{\circ}\right)\right)^{o}$, where the superscript " $O$ " means the opposite category.

1.3. Suppose now that $\mathcal{C}$ is an additive (resp., $\mathbb{C}$-linear) category. Then every object $F$ of $\operatorname{Ind}(\mathcal{C})$, which is a priori a contravariant functor $\mathcal{C} \rightarrow$ Set, lifts in a natural way to an additive functor $\mathcal{C} \rightarrow\{$ Abelian groups $\}$ (resp., $\mathbb{C}$-linear functor $\mathcal{C} \rightarrow V e c t$ ).

Indeed if for some $X_{i} \in \mathcal{C}, X=$ "lim" $X_{i}$, for the corresponding Hom sets we have: $X(Y)=$ $\underset{\lim }{\longrightarrow} \operatorname{Hom}\left(Y, X_{i}\right)$, and this inductive limit of sets has a natural structure of an abelian group

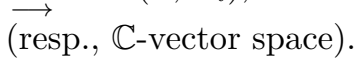

Suppose now that $\mathcal{C}$ is abelian. We will now give a simple criterion that establishes indrepresentability of functors in this case. Together with Lemma 1.2 this provides a tool to prove representability of various functors in the framework of abelian categories.

Assume that $\mathcal{C}$ is such that for a given object the class if its subobjects is a set. Let $F$ : $\mathrm{e} \rightarrow\{$ Abelian groups $\}$ be a contravariant left exact functor. Suppose that there exists another functor $F^{\prime}$ and a morphism of functors $F \rightarrow F^{\prime}$ such that $\forall X \in \mathcal{C}$ the map $F(X) \rightarrow F^{\prime}(X)$ is injective.

Proposition 1.4. Assume that for $\mathcal{C}, F$ and $F^{\prime}$ as above, the functor $F^{\prime}$ is ind-representable. Then the functor $F$ is also ind-representable.

Proof. (Compare 2], Corollary 2.8)

Let $Z=$ "lim" $Z_{i}$ be the object of Ind(C) ind-representing $F^{\prime}$. For each index $i$ consider the functor $F_{i}$ equal to $F \underset{F^{\prime}}{\times} \operatorname{Hom}\left(\cdot, Z_{i}\right)$. Each $F_{i}$ is also left exact and its map to $\operatorname{Hom}\left(\cdot, Z_{i}\right)$ is an injection. Obviously, $F(Y)=\underset{\lim }{\longrightarrow} F_{i}(Y)$, so it enough to show that each $F_{i}$ is ind-representable. In other words, we can assume that $F^{\prime}$ is representable by an object $Z \in \mathfrak{C}$.

Consider the category of pairs $(X \in \mathcal{C}, \alpha: X \rightarrow Z)$, where $\alpha$ is an injective morphism in $\mathcal{C}$ such that the corresponding element in $F^{\prime}(X)$ belongs to $F(X)$. (Morphisms between $(X \in \mathcal{C}, \alpha: X \rightarrow Z)$ and $\left(X^{\prime} \in \mathcal{C}, \alpha^{\prime}: X^{\prime} \rightarrow Z\right)$ are maps $X \rightarrow X^{\prime}$ in $\mathcal{C}$, which commute with the data of $\alpha$ and $\alpha^{\prime}$.) This category is obviously discrete, and it is small due to our assumption on $\mathcal{C}$. This resulting poset is filtering and is endowed with a functor to $\mathcal{C}$, i.e., $(X \in \mathcal{E}, \alpha: X \rightarrow Z) \mapsto X$. 
Let $W \in \operatorname{Ind}(\mathcal{C})$ be the direct limit of this system. We claim that $W$ ind-represents the functor $F$. Indeed, for $Y \in \mathcal{C}$, given an element in $\operatorname{Hom}(Y, W)$ we have for some $X$ an element in $F(X)$ and a map $Y \rightarrow X$, which gives rise to an element of $F(Y)$.

And vice versa, given an element in $a_{Y} \in F(Y)$ consider the corresponding element $a_{Y}^{\prime} \in$ $F^{\prime}(Y)$ and the resulting map $Y \rightarrow Z$. Let $X$ be the image of this map:

$$
Y \rightarrow X \hookrightarrow Z
$$

It is enough to show that $a_{Y}$ belongs to the image of $F(X)$. Since $F$ is left exact, it is enough to show that the image of $a_{Y}$ vanishes in $F(\operatorname{ker}(Y \rightarrow X))$. However, by assumption, the image of $a_{Y}^{\prime}$ in $F^{\prime}(\operatorname{ker}(Y \rightarrow X))$ is zero, which implies our assertion, since $F \rightarrow F^{\prime}$ is injective.

The following is also well-known (cf. 2], Proposition 4.5):

Lemma 1.5. If $\mathcal{C}$ is abelian, then so is $\operatorname{Ind}(\mathcal{C})$. The functor limInd $: \operatorname{Ind}(\operatorname{Ind}(\mathcal{C})) \rightarrow \operatorname{Ind}(\mathcal{C})$ is exact.

Of course, assertions similar to the above ones hold when we replace Ind by Pro.

1.6. The following category will play an essential role in this paper:

$$
\mathbb{V} e c t:=\operatorname{Pro}(\text { Vect }) \simeq \operatorname{Pro}\left(\operatorname{Ind}\left(\text { Vect }_{0}\right)\right) .
$$

According to Lemma 1.5 this is an abelian category.

We will also consider the categories $\mathbf{S e t}:=\operatorname{Ind}\left(\operatorname{Pro}\left(\operatorname{Set}_{0}\right)\right)$, and

$$
\text { Set }:=\operatorname{Ind}(\operatorname{Pro}(\mathbf{S e t})) \simeq \operatorname{Ind}\left(\operatorname{Pro}\left(\operatorname{Ind}\left(\operatorname{Pro}\left(\text { Set }_{0}\right)\right)\right)\right)
$$

where $S_{e} t_{0}$ is the category of finite sets.

Note that the category $\operatorname{Pro}\left(\operatorname{Set}_{0}\right)$ is equivalent to the category of compact totally disconnected topological spaces; let us denote this equivalence by $\mathbf{Y} \mapsto \mathbf{Y}^{\text {top }}$. If $\mathbf{Y}=$ "lim" $Y_{j}$, then $\mathbf{Y}^{\text {top }} \simeq \mathbf{Y}=\lim Y_{j}$, where the projective limit is taken in the category of topological spaces. For $\mathbf{X} \in \mathbf{S}$ et presented as a direct limit "lim" $\mathbf{X}_{i}$ with $\mathbf{X}_{i} \in \operatorname{Pro}\left(\operatorname{Set}_{0}\right)$, set $\mathbf{X}^{\text {top }}$ to be the topological space $\lim \mathbf{X}_{i}^{\text {top }}$ (where the inductive limit is again taken in the category of topological spaces).

We will use the following terminology. We will call an object $\mathbf{X} \in \mathbf{S}$ et compact, if it belongs to $\operatorname{Pro}\left(\operatorname{Set}_{0}\right)$, and a morphism $\mathbf{X} \rightarrow \mathbf{Y}$ in $\mathbf{S} e t$ proper if every base change by a compact object is compact.

We will call an object $\mathbf{X} \in \mathbf{S} e t$ locally compact, if it can be represented as a direct limit $\mathbf{X}=$ "lim" $\mathbf{X}_{i}, \mathbf{X}_{i} \in \operatorname{Pro}\left(\right.$ Set $\left._{0}\right)$, where the maps $\mathbf{X}_{i} \rightarrow \mathbf{X}_{j}$ are such that the corresponding maps of topological spaces $\mathbf{X}_{i}^{\text {top }} \rightarrow \mathbf{X}_{j}^{\text {top }}$ are open embeddings.

The full subcategory of $\mathbf{S e t}$ consisting of locally compact objects is equivalent to the category Top ${ }^{\text {Hlctd }}$ of Hausdorff locally compact totally disconnected topological spaces. All objects of Set that are relevant for the purposes of this paper will be locally compact. Therefore, the reader may safely replace $\mathbf{S e t}$ by $T o p^{H l c t d}$ and $\operatorname{Set}$ by $\operatorname{Ind}\left(\operatorname{Pro}\left(T o p^{H l c t d}\right)\right)$.

Similarly, we will call an object $\mathbb{X} \in \mathbb{S e t}$ bounded if it actually belongs to $\operatorname{Pro}(\mathbf{S e t})$. 
1.7. Let $\mathcal{A}$ be a monoidal category, i.e., we have a functor $\otimes: \mathcal{A} \times \mathcal{A} \rightarrow \mathcal{A}$, a unit object $\mathbf{1}_{\mathcal{A}} \in \mathcal{A}$ and functorial isomorphisms

$$
X \otimes(Y \otimes Z) \simeq(X \otimes Y) \otimes Z ; \quad X \otimes \mathbf{1}_{\mathcal{A}} \simeq X \simeq \mathbf{1}_{\mathcal{A}} \otimes X,
$$

obeying the usual axioms. Note that in this case the categories $\operatorname{Ind}(\mathcal{A})$ and $\operatorname{Pro}(\mathcal{A})$ also possess natural monoidal structures.

If $\mathcal{C}$ is another category, there is a standard notion of action of $\mathcal{A}$ on $\mathcal{C}$, in which case we say that $\mathcal{C}$ is a module category over $\mathcal{A}$. Namely, a module structure is a functor $\otimes: \mathcal{A} \times \mathcal{C} \rightarrow \mathcal{C}$, and for $X, Y \in \mathcal{A}$ and $V \in \mathcal{C}$ functorial isomorphisms

$$
(X \otimes Y) \otimes V \rightarrow X \otimes(Y \otimes V) ; \mathbf{1}_{\mathcal{A}} \otimes V \simeq V,
$$

satisfying the natural axioms. In particular, for $X \in \mathcal{A}, V, W \in \mathcal{C}$ we have a well-defined Hom set $\operatorname{Hom}(X \otimes V, W)$.

By definition, a pseudo-action of $\mathcal{A}$ on $\mathcal{C}$ (or a structure on $\mathcal{C}$ of a pseudo-module over $\mathcal{A}$ ) is a functor $\mathcal{A}^{o} \times \mathcal{C}^{o} \times \mathcal{C} \rightarrow$ Set, denoted $\mathcal{H} o m(\cdot \otimes \cdot, \cdot)$, and a morphism of functors: for $X, Y \in \mathcal{A}$, $V, U, W \in \mathcal{C}$

$$
\mathcal{H} \circ \mathrm{m}(X \otimes V, W) \times \mathcal{H} \text { om }(Y \otimes U, V) \Rightarrow \mathcal{H} \text { om }((X \otimes Y) \otimes U, W),
$$

and a functorial isomorphism $\mathcal{H} \operatorname{om}\left(\mathbf{1}_{\mathcal{A}} \otimes V, W\right) \simeq \operatorname{Hom}_{\mathcal{C}}(V, W)$, such that the following compatibility conditions hold:

For $X, Y, Z \in \mathcal{A}, V, U, W, Q \in \mathcal{C}$, the arrows

$$
\begin{aligned}
& \mathcal{H} \operatorname{Hom}(X \otimes W, Q) \times \mathcal{H o m}(Y \otimes V, W) \times \mathcal{H} \text { om }(Z \otimes U, V) \rightarrow \\
& \rightarrow \mathcal{H} \text { om }(X \otimes W, Q) \times \mathcal{H o m}((Y \otimes Z) \otimes U, W) \rightarrow \mathcal{H} \text { om }((X \otimes(Y \otimes Z)) \otimes U, Q) \text { and } \\
& \mathcal{H} \operatorname{Hom}(X \otimes W, Q) \times \mathcal{H} \text { om }(Y \otimes V, W) \times \mathcal{H} \text { om }(Z \otimes U, V) \rightarrow \\
& \rightarrow \mathcal{H} \text { om }((X \otimes Y) \otimes V, Q) \otimes \mathcal{H} \text { om }(Z \otimes U, V) \rightarrow \mathcal{H} \text { om }(((X \otimes Y) \otimes Z) \otimes U, Q)
\end{aligned}
$$

coincide under the associativity isomorphism $(X \otimes Y) \otimes Z \simeq X \otimes(Y \otimes Z)$, and for $U, V, W \in \mathcal{C}$ and $X \in \mathcal{A}$, the squares

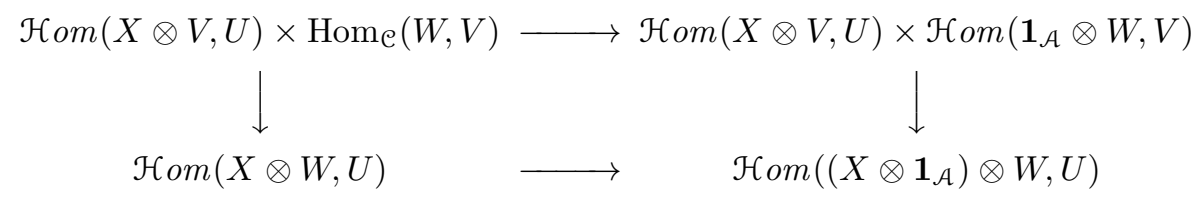

and

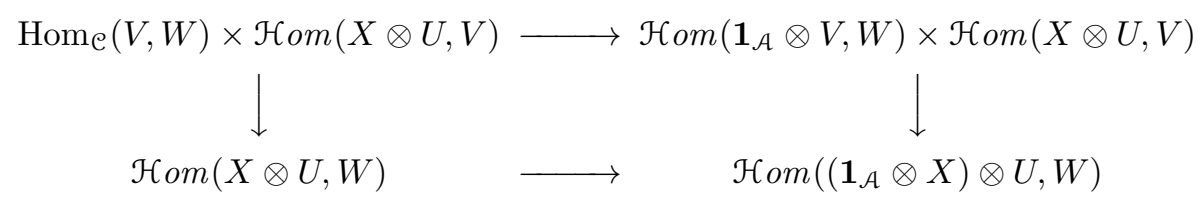

are commutative.

Note that if $\mathcal{C}$ is a pseudo-module over $\mathcal{A}$, then $\mathcal{C}^{o}$ is a pseudo-module over $\mathcal{A}^{o p}$, where the latter is the category $\mathcal{A}$ with the opposite monoidal structure:

$$
\mathcal{H} \operatorname{com}\left(X \otimes V^{o}, W^{o}\right):=\mathcal{H} \operatorname{com}(X \otimes W, V) .
$$

When $\mathcal{C}$ is additive (resp., $\mathbb{C}$-linear), we will rather use the variant of the above definition, when we require that the sets $\mathcal{H} \operatorname{om}(X \otimes U, V)$ have a structure of an abelian group (resp., $\mathbb{C}$-vector space), such that the natural transformations $\mathcal{H}$ om $(X \otimes U, V) \times \mathcal{H}$ om $(Y \otimes V, W) \Rightarrow$

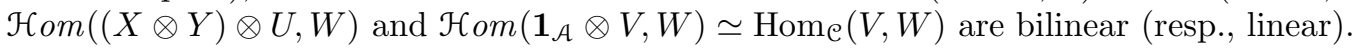


For example, the category Set is a monoidal via $X \otimes Y:=X \times Y$, and any category $\mathcal{C}$ has a pseudo-module structure over $\operatorname{Set}$ via $\mathcal{H} o m(X \otimes U, V):=\operatorname{Hom}(U, V)^{X}$ for $X \in S e t, U, V \in \mathcal{C}$.

Of course, when $\mathcal{C}$ is a module category over $\mathcal{A}$, it acquires a pseudo-module structure by setting

$$
\mathcal{H} o m(X \otimes U, V):=\operatorname{Hom}_{\mathcal{C}}(X \otimes U, V) .
$$

In what follows we will say that an element $\phi \in \mathcal{H} o m(X \otimes U, V)$ defines an action $X \times U \rightarrow V$.

1.8. Let us now analyze how pseudo-actions behave when we Ind- and Pro- complete our categories.

First, we claim that if $\mathcal{A}$ pseudo-acts on $\mathcal{C}$, then so do $\operatorname{Ind}(\mathcal{A})$ and $\operatorname{Pro}(\mathcal{A})$. Indeed, if $X \in \operatorname{Ind}(\mathcal{A})$ (resp., $X \in \operatorname{Pro}(\mathcal{A}))$ is "lim" $X_{i}$ (resp., "lim" $X_{i}$ ), we set $\mathcal{H}$ om $(X \otimes V, W)=$ $\stackrel{\lim }{\leftarrow} \mathcal{H o m}\left(X_{i} \otimes V, W\right)$ (resp., $\left.\mathcal{H o m}(X \otimes \vec{V}, W)=\underline{\lim } \mathcal{H} \overleftarrow{\operatorname{Hom}}\left(X_{i} \otimes V, W\right)\right)$. It is easy to see that this definition is independent of the way we represent $X$ as a direct (resp., inverse) limit.

Also, if $\mathcal{C}$ has a pseudo-module structure over $\mathcal{A}$, so do $\operatorname{Ind}(\mathcal{C})$ and $\operatorname{Pro}(\mathcal{C})$. Indeed, for $V, W \in$ $\operatorname{Ind}(\mathcal{C})$ equal to "lim" $V_{i}$ and "lim" $W_{j}$, (resp., "lim" $V_{i}$ and "lim" $\left.W_{j}\right)$, we set $\mathcal{H} o m(X \otimes V, W)$ to be

$$
\left.\left.\underset{i}{(\lim }) \underset{j}{(\lim }) \mathcal{H} \operatorname{Hom}\left(X \otimes V_{i}, W_{j}\right) \text { and } \underset{j}{(\lim }\right) \underset{i}{(\lim }\right) \mathcal{H} \operatorname{Hom}\left(X \otimes V_{i}, W_{j}\right)
$$

respectively. One can easily see that this definition is independent of the presentation of $V$ and $W$ as directs (resp., inverse) limits.

Now, we obtain that there are two pseudo-actions of $\operatorname{Ind}(\mathcal{A})$ on $\operatorname{Ind}(\mathcal{C})$. One is (which we will call "naive") when we first consider the pseudo-action of $\operatorname{Ind}(\mathcal{A})$ on $\mathcal{C}$ and then produce from it the corresponding pseudo-action on $\operatorname{Ind}(\mathcal{C})$. The other is when we first consider the pseudo-action of $\mathcal{A}$ on $\operatorname{Ind}(\mathcal{C})$ and then produce from it the corresponding pseudo-action of $\operatorname{Ind}(\mathcal{A})$. Unless specified otherwise, in the sequel we will use the pseudo-action of the second kind. Note that we have a canonical map $\mathcal{H} o m(X \otimes V, W)_{\text {naive }} \rightarrow \mathcal{H}$ om $(X \otimes V, W)$. In concrete

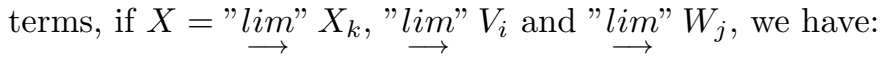

$$
\begin{aligned}
& \left.\left.\mathcal{H o m}(X \otimes V, W)_{\text {naive }}=\left(\overleftarrow{i}_{i} \underset{j}{\lim }\right) \underset{k}{(\lim }\right) \underset{(\lim }{(\lim }\right) \mathcal{H} \operatorname{Hom}\left(X_{k} \otimes V_{i}, W_{j}\right) ; \\
& \mathcal{H} \operatorname{Hom}(X \otimes V, W)=(\underbrace{\lim }_{k}) \underset{i}{(\lim }) \underset{j}{(\lim }) \mathcal{H} \operatorname{Hom}\left(X_{k} \otimes V_{i}, W_{j}\right)
\end{aligned}
$$

For example, by taking $\mathcal{C}=\mathcal{A}$, the canonical action of $\operatorname{Ind}(\mathcal{A})$ on itself corresponding to the monoidal structure coincides with the pseudo-action described above coming from the action on $\mathcal{A}$ on itself.

Similarly, we obtain the corresponding notions concerning the pseudo-action of $\operatorname{Ind}(\mathcal{A})$ on $\operatorname{Pro}(\mathcal{C})$.

The situation with the pseudo-actions of $\operatorname{Pro}(\mathcal{A})$ is the opposite. The naive pseudo-module structure on $\operatorname{Ind}(\mathcal{C})$ is obtained when we first consider the pseudo-action of $\mathcal{A}$ on $\operatorname{Ind}(\mathcal{C})$, and then produce from it a pseudo-action of $\operatorname{Pro}(\mathcal{A})$. The pseudo-module structure that we will normally consider is is obtained by first considering the pseudo-action of $\operatorname{Pro}(\mathcal{A})$ on $\mathcal{C}$, and then producing from it the corresponding pseudo-action on $\operatorname{Ind}(\mathcal{C})$. As before, we have a canonical map $\mathcal{H o m}(X \otimes V, W)_{\text {naive }} \rightarrow \mathcal{H}$ om $(X \otimes V, W)$, and for $V=$ "lim" $V_{i}, W=$ "lim" $W_{j}$ and 
$X=" \underset{\leftarrow}{\lim } " X_{k}$

$$
\begin{aligned}
& \left.\left.\left.\mathcal{H} \text { om }(X \otimes V, W)_{\text {naive }}=\underset{k}{(\lim }\right) \underset{i}{(\lim }\right) \underset{j}{(\lim }\right) \mathcal{H} \operatorname{Hom}\left(X_{k} \otimes V_{i}, W_{j}\right) ;
\end{aligned}
$$

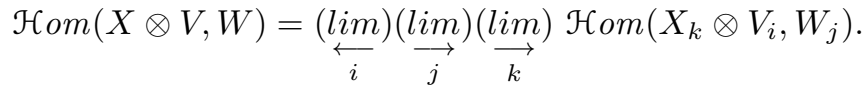

In a similar way, we obtain the two pseudo-actions of $\operatorname{Pro}(\mathcal{A})$ on $\operatorname{Pro}(\mathcal{C})$. As above, for $\mathcal{C}=\mathcal{A}$ this canonical pseudo-action coincides with the action corresponding to the monoidal structure on $\operatorname{Pro}(\mathcal{A})$.

Finally, we see that there are 3 possible pseudo-actions of $\operatorname{Ind} \operatorname{Pro}(\mathcal{A})$ on $\operatorname{Ind}(\mathcal{C})$. The one that we will consider is "the biggest": we will first consider the pseudo-action of $\operatorname{Pro}(\mathcal{A})$ on $\mathcal{C}$, then produce from it the pseudo-action of $\operatorname{Pro}(\mathcal{A})$ on $\operatorname{Ind}(\mathcal{C})$, and then the pseudo-action of $\operatorname{Ind} \operatorname{Pro}(\mathcal{A})$ on $\operatorname{Ind}(\mathcal{C})$.

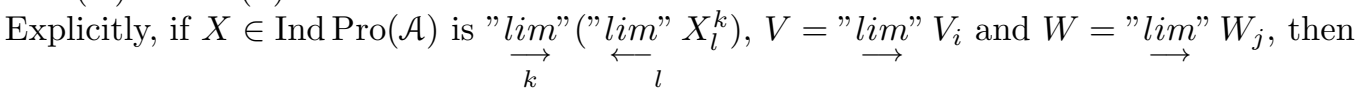

$$
\mathcal{H} o m(X \otimes V, W)=\overleftarrow{k}_{k}^{\lim } \underset{i}{\lim } \underset{l}{\lim } \underset{j}{\lim } \mathcal{H} \operatorname{Hom}\left(X_{l}^{k} \otimes V_{i}, W_{j}\right)
$$

By inverting the arrows in $\mathcal{C}$ we obtain the corresponding pseudo-action of $\operatorname{Ind} \operatorname{Pro}(\mathcal{A})$ on $\operatorname{Pro}(\mathcal{C})$.

1.9. Let us consider our main examples. Let $\mathcal{A}=S_{e t}$, and $\mathcal{C}=V e c t_{0}$. Then for $\mathbf{X} \in \mathbf{S e t}=$ $\operatorname{Ind}\left(\operatorname{Pro}\left(\right.\right.$ Set $\left.\left._{0}\right)\right), \mathbf{V}, \mathbf{W} \in V e c t=\operatorname{Ind}\left(\right.$ Vect $\left._{0}\right)$, we obtain the notion of an action $\mathbf{X} \times \mathbf{V} \rightarrow \mathbf{W}$. However, it is easy to see that such an action is the same as a continuous map $\mathbf{X}^{\text {top }} \times \mathbf{V} \rightarrow \mathbf{W}$, linear in $\mathbf{V}$ and $\mathbf{W}$, where $\mathbf{V}$ and $\mathbf{W}$ are endowed with the discrete topology, and $\mathbf{X}^{\text {top }}$ is as in Sect. 1.6.

Now set $\mathcal{A}=\mathbf{S} e t=\operatorname{Ind}\left(\operatorname{Pro}\left(\operatorname{Set}_{0}\right)\right)$ and $\mathcal{C}=V e c t$. We obtain a pseudo-module structure on $\mathbb{V} e c t$ with respect to Set.

Let us write down the last notion in more concrete terms. First, let $\mathbb{X}$ be an object of $\operatorname{Pro}(\mathbf{S} e t)$, and $\mathbb{V}, \mathbb{W}$ be two objects of $\mathbb{V} e c t$. An action $\phi: \mathbb{X} \times \mathbb{V} \rightarrow \mathbb{W}$ is the following data. Let $\mathbb{X}="$ lim" $\mathbf{X}_{j}, \mathbb{V}=" l i m " \mathbf{V}_{i}, \mathbb{W}=" l i m " \mathbf{W}_{i^{\prime}}$, with $\mathbf{X}_{j} \in \mathbf{S e t}, \mathbf{V}_{i}, \mathbf{W}_{i^{\prime}} \in$ Vect. Then for every $i^{\prime}$ there must exist $i_{0}, j_{0}$ and a compatible system of action maps $\phi_{j, i, i^{\prime}}: \mathbf{X}_{j} \times \mathbf{V}_{i} \rightarrow \mathbf{W}_{i^{\prime}}$ defined for $i \geq i_{0}, j \geq j_{0}$. Another compatibility condition is imposed: for $i_{1}^{\prime} \geq i_{2}^{\prime}$ the corresponding diagrams

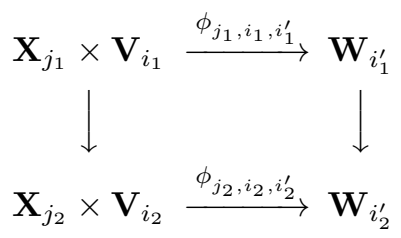

must commute for $i_{1}$ and $j_{1}$ large enough. Two action maps $\phi$ and $\psi$ coincide if for every $i^{\prime}$ the corresponding maps $\phi_{j, i, i^{\prime}}$ and $\psi_{j, i, i^{\prime}}$ coincide for $i$ and $j$ large enough.

If now $\mathbb{X}$ is an object of Set, equal to "lim" $\mathbb{X}^{j}$ and $\mathbb{V}, \mathbb{W} \in \mathbb{V}$ ect, an action $\phi: \mathbb{X} \times \mathbb{V} \rightarrow \mathbb{W}$ is a compatible system of actions $\phi^{j}: \mathbb{X}^{j} \times \overrightarrow{\mathbb{V}} \rightarrow \mathbb{W}$. 
1.10. The following definition will be needed in the sequel. First, note that we have an obvious functor from the category of sets (denoted Set) to Set via

$$
S e t \simeq \operatorname{Ind}\left(\operatorname{Set}_{0}\right) \rightarrow \operatorname{Ind}\left(\operatorname{Pro}\left(\operatorname{Set}_{0}\right)\right) \simeq \mathbf{S e t} .
$$

Let $\mathbf{X}_{1} \rightarrow \mathbf{X}_{2}$ be a map of objects of $\mathbf{S} e t$. We will say that it is weakly surjective if for any $Y \in$ Set, the map

is injective.

$$
\operatorname{Hom}_{\mathbf{S} e t}\left(\mathbf{X}_{2}, Y\right) \rightarrow \operatorname{Hom}_{\mathbf{S e t}}\left(\mathbf{X}_{1}, Y\right)
$$

Note that if $\mathbf{X}_{1}, \mathbf{X}_{2}$ are locally compact, the above notion that a morphism $\mathbf{X}_{1} \rightarrow \mathbf{X}_{2}$ is weakly surjective is equivalent to the condition that the corresponding map $\mathbf{X}_{1}^{\text {top }} \rightarrow \mathbf{X}_{2}^{\text {top }}$ has dense image.

Lemma 1.11. A map $\mathbf{X}_{1} \rightarrow \mathbf{X}_{2}$ in $\mathbf{S} e t$ is weakly surjective if and only if for any $\mathbf{V}, \mathbf{W} \in V e c t$, the map $\mathcal{H} o m\left(\mathbf{X}_{2} \otimes \mathbf{V}, \mathbf{W}\right) \rightarrow \mathcal{H} \operatorname{Hom}\left(\mathbf{X}_{1} \otimes \mathbf{V}, \mathbf{W}\right)$ is injective.

We will call an object $\mathbb{X} \in \operatorname{Pro}(\mathbf{S} e t)$ weakly strict if it can be represented as "lim" $\mathbf{X}_{i}$, where the maps $\mathbf{X}_{j} \rightarrow \mathbf{X}_{i}$ are weakly surjective.

Note that if $\mathbb{X}$ is weakly strict and $\mathbf{V}, \mathbf{W} \in V$ ect, for any element $\phi \in \mathcal{H} \operatorname{com}(\mathbb{X} \otimes \mathbf{V}, \mathbf{W})$ we have well-defined kernel and image of $\phi$. By definition, $\operatorname{ker}(\phi) \subset \mathbf{V}(\operatorname{resp} ., \operatorname{Im}(\phi) \subset \mathbf{W})$ is the maximal (resp., minimal) subspace $\mathbf{V}^{\prime}$ of $\mathbf{V}$ (resp., $\mathbf{W}^{\prime}$ of $\mathbf{W}$ ) having the property that $\phi$ factors through an element $\phi^{\prime} \in \mathcal{H} \circ \mathrm{om}\left(\mathbb{X} \otimes \mathbf{V} / \mathbf{V}^{\prime}, \mathbf{W}^{\prime}\right)\left(\right.$ resp., $\left.\phi^{\prime} \in \mathcal{H} \circ \mathrm{m}\left(\mathbb{X} \otimes \mathbf{V}, \mathbf{W}^{\prime}\right)\right)$.

Indeed, both $\operatorname{ker}(\phi)$ and $\operatorname{Im}(\phi)$ are clearly well-defined when $\mathbf{X} \in \mathbf{S}$ et. If now $\mathbb{X}=" l i m " \mathbf{X}_{i}$, with weakly surjective maps, and $\phi$ comes from an element $\phi_{i} \in \mathcal{H} \circ \mathrm{m}\left(\mathbf{X}_{i} \otimes \mathbf{V}, \mathbf{W}\right)$, then it is easy to see that $\operatorname{ker}\left(\phi_{i}\right) \subset \mathbf{V}$ and $\operatorname{Im}\left(\phi_{i}\right) \subset \mathbf{W}$ are the sought-for subspaces.

\section{Categories of representations}

2.1. In the abstract set-up of the previous section, let us recall that an object $X \in \mathcal{A}$ is called a monoid (in the sense of the monoidal structure on $\mathcal{A}$ ) if we are given a (multiplication) map $X \otimes X \rightarrow X$ and a (unit) map $\mathbf{1}_{\mathcal{A}} \rightarrow X$, which satisfy the usual associativity and unit axioms.

In our examples, the monoidal structure on $\mathcal{A}$ will be such that $X \otimes Y$ is isomorphic to the categorical direct product $X \times Y$. Moreover, $\operatorname{Hom} \mathcal{C}\left(X, \mathbf{1}_{\mathcal{A}}\right)$ will be a one-element set $\forall X \in \mathcal{C}$. Note that this property is inherited by both $\operatorname{Ind}(\mathcal{A})$ and $\operatorname{Pro}(\mathcal{A})$.

In this case, it makes sense to speak about group-objects in $\mathcal{A}$ : a monoid $X$ is called a group if there exists a map $\gamma: X \rightarrow X$ (automatically unique) such that the two compositions

$$
\begin{gathered}
X \stackrel{\Delta}{\rightarrow} X \times X \stackrel{\text { id } \times \gamma}{\longrightarrow} X \times X \stackrel{\text { mult }}{\longrightarrow} X \text { and } \\
X \stackrel{\Delta}{\rightarrow} X \times X \stackrel{\gamma \times \text { id }}{\longrightarrow} X \times X \stackrel{\text { mult }}{\longrightarrow} X
\end{gathered}
$$

are both equal to $X \rightarrow \mathbf{1}_{\mathcal{A}} \rightarrow X$.

In the sequel we will only consider monoids, which are groups.

2.2. If $\mathcal{C}$ is another category with a pseudo-action of $\mathcal{A}$ and $X \in \mathcal{A}$ is a monoid, a representation of $X$ in $\mathcal{C}$ is a pair $\Pi=(V, \rho)$, where $V \in \mathcal{C}$ and $\rho \in \mathcal{H} \operatorname{oom}(X \otimes V, V)$, such that the following two conditions hold:

Associativity: The image of $\rho \times \rho$ under the associativity constraint

$$
\mathcal{H} o m(X \otimes V, V) \otimes \mathcal{H} \text { om }(X \otimes V, V) \rightarrow \mathcal{H} \text { om }((X \otimes X) \otimes V, V)
$$

equals the image of $\rho$ under the map $\mathcal{H} o m(X \otimes V, V) \rightarrow \mathcal{H}$ om $(X \otimes X) \otimes V, V)$ given by the multiplication $X \otimes X \rightarrow X$. 
Unit: The image of $\rho$ in $\mathcal{H} \operatorname{oom}\left(\mathbf{1}_{\mathcal{A}} \otimes V, V\right)$ under $\mathbf{1}_{\mathcal{A}} \rightarrow X$ equals the identity element in $\mathcal{H} o m\left(\mathbf{1}_{\mathcal{A}} \otimes V, V\right) \simeq \operatorname{Hom}(V, V)$.

Representations of $X$ in $\mathcal{C}$ form a category, which we will denote by $\operatorname{Rep}(X, \mathcal{C})$. When $\mathcal{C}$ is additive (resp., $\mathbb{C}$-linear), the category $\operatorname{Rep}(X, \mathcal{C})$ is additive (resp., $\mathbb{C}$-linear) as well.

Assume now that $\mathcal{C}$ is abelian and that for a fixed $X \in \mathcal{A}$, the functor $\mathcal{C}^{\circ} \times \mathcal{C} \rightarrow$ Set given by $V, W \mapsto \mathcal{H} o m(X \otimes V, W)$ is left-exact in both arguments.

Lemma 2.3. Under the above circumstances the category $\operatorname{Rep}(X, \mathcal{C})$ is abelian and the natural forgetful functor $\operatorname{Rep}(X, \mathcal{C}) \rightarrow \mathcal{C}$ is exact.

If $\mathcal{A}$ is a monoidal category with a pseudo-action on an abelian category $\mathcal{C}$, such that the above left-exactness condition is satisfied, then the same holds for $\operatorname{Ind}(\mathcal{A})(\operatorname{resp} ., \operatorname{Pro}(\mathcal{A})$ ) pseudoacting on $\operatorname{Ind}(\mathcal{C})$ (resp., Pro(e)), due to the fact that the functor limInd (resp., limProj) is exact (resp., left-exact) on the category of abelian groups.

In particular, we obtain that this condition is satisfied in our examples of $\mathcal{A}=\mathbf{S} e t, \mathcal{C}=V e c t$ and $\mathcal{A}=\operatorname{Set}, \mathcal{C}=\mathbb{V e c t}$.

2.4. Set first $\mathcal{A}=\mathbf{S e t}$, and $\mathcal{C}=$ Vect. Thus, for a group-object $\mathbf{H} \in \mathbf{S}$ et the category $\operatorname{Rep}(\mathbf{H}, V e c t)$ is the usual category of representations of $\mathbf{H}$ appearing in the theory of $\mathfrak{p}$-adic groups. In other words, if $\mathbf{H}$ is locally compact (cf. Sect. 1.6) and $\mathbf{H}^{\text {top }}$ is the corresponding topological group, then an object of $\operatorname{Rep}(\mathbf{H}, V e c t)$ is the same as a smooth representation of $\mathbf{H}^{\text {top }}$.

If $\mathbb{H}$ is a group-object of $\operatorname{Pro}(\mathbf{S} e t)$, we can consider its representations on Vect and $\mathbb{V} e c t$, and the resulting categories will be denoted by $\operatorname{Rep}(\mathbb{H}, V e c t)$ and $\operatorname{Rep}(\mathbb{H}, \mathbb{V} e c t)$, respectively.

We will say that $\mathbb{H} \in \operatorname{Pro}(\mathbf{S e t})$ satisfies condition $(*)$ if it is weakly strict as an object of $\operatorname{Pro}($ Set $)$, cf. Sect. 1.10

The following assertion will play an important role in the sequel: ${ }^{1}$

Proposition 2.5. For $\mathbb{H}$ satisfying (*), the categories $\operatorname{Rep}(\mathbb{H}, \mathbb{V}$ ect $)$ and $\operatorname{Pro}(\operatorname{Rep}(\mathbb{H}$, Vect $))$ are naturally equivalent.

Note that the proof given below is valid when $\mathbb{H}$ is a just a monoid (not necessarily a group), satisfying condition $(*)$.

Proof. The functor in one direction: $\mathrm{F}: \operatorname{Pro} \operatorname{Rep}(\mathbb{H}, V e c t)) \rightarrow \operatorname{Rep}(\mathbb{H}, \mathbb{V e c t})$ is evident; moreover, it is easy to see that it is fully faithful. Let us show that it admits a left adjoint.

For $(\mathbb{V}, \rho) \in \operatorname{Rep}(\mathbb{H}, \mathbb{V} e c t)$, let us write $\mathbb{V}=" l i m " \mathbf{V}_{i}$, where the index $i$ runs over some filtering set $I$. Consider the category of quadruples $\left(\mathbf{V}^{\prime}, \rho^{\prime}, i, \alpha: \mathbf{V}_{i} \rightarrow \mathbf{V}^{\prime}\right)$, where $\left(\mathbf{V}^{\prime}, \rho^{\prime}\right) \in$ $\operatorname{Rep}(\mathbb{H}, V e c t), i \in I$ and $\alpha$ is a map, such that its image generates $\mathbf{V}^{\prime}$ as an $\mathbb{H}$-representation and the composition $\mathbb{V} \rightarrow \mathbf{V}_{i} \rightarrow \mathbf{V}^{\prime}$ is compatible with the $\mathbb{H}$-actions. A morphism between $\left(\mathbf{V}_{1}, \rho_{1}^{\prime}, i_{1}, \alpha_{1}: \mathbf{V}_{i_{1}} \rightarrow \mathbf{V}_{1}^{\prime}\right)$ and $\left(\mathbf{V}_{2}, \rho_{2}^{\prime}, i_{2}, \alpha_{2}: \mathbf{V}_{i_{2}} \rightarrow \mathbf{V}_{2}^{\prime}\right)$ is by definition a relation $i_{2} \geq i_{1}$ and a map of $\mathbb{H}$-representations $\mathbf{V}_{1}^{\prime} \rightarrow \mathbf{V}_{2}^{\prime}$, such that the square

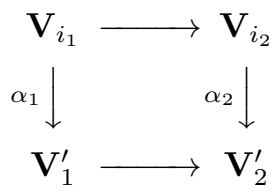

\footnotetext{
${ }^{1}$ We would like to thank E. Hrushovski for pointing out the mistake in the previous version of the paper, where Proposition 2.5 was stated without the $(*)$ assumption on $H$; in fact, he constructed a counterexample.
} 
commutes. The resulting category is evidently discrete, filtering and small. By definition, we have a forgetful functor from this category to $\operatorname{Rep}(\mathbb{H}, V e c t)$ that sends a quadruple $\left(\mathbf{V}^{\prime}, \rho^{\prime}, i, \alpha\right)$ to $\left(\mathbf{V}^{\prime}, \rho^{\prime}\right)$. Let us denote by $\mathrm{G}(\mathbb{V}, \rho) \in \operatorname{Pro}(\operatorname{Rep}(\mathbb{H}, V e c t))$ the resulting inverse limit. It is easy too see that the assignment $(\mathbb{V}, \rho) \mapsto \mathrm{G}(\mathbb{V}, \rho)$ defines a functor left adjoint to $\mathrm{F}$.

The fact that $F$ was fully-faifull means that the composition $G \circ F$ is isomorphic to the identity functor. Thus, it remains to see that for $(\mathbb{V}, \rho) \in \operatorname{Rep}(\mathbb{H}, \mathbb{V} e c t)$, the adjunction map $(\mathbb{V}, \rho) \rightarrow \mathrm{F} \circ \mathrm{G}(\mathbb{V}, \rho)$ is an isomorphism. For that, it suffices to show that if $\mathbb{V}=$ "lim" $\mathbf{V}_{i}$, then for every $i$ there exists a vector space $\mathbf{V}_{i}^{\prime}$ underlying an object $\Pi=\left(\mathbf{V}_{i}^{\prime}, \rho_{i}\right) \in \operatorname{Rep}(\mathbb{H}, V e c t)$, such that the map $\mathbb{V} \rightarrow \mathbf{V}_{i}$ factors as $\mathbb{V} \rightarrow \mathbf{V}_{i}^{\prime} \rightarrow \mathbf{V}_{i}$, with the first arrow preserving the $\mathbb{H}$-action. Indeed, this would show that the map $(\mathbb{V}, \rho) \rightarrow \mathrm{F} \circ \mathrm{G}(\mathbb{V}, \rho)$ is always injective, and combined with the fact that $\mathrm{G}$ is right-exact, this implies that this map is an isomorphism.

Let $j$ be an index such that the map $\mathbb{H} \times \mathbb{V} \stackrel{\text { act }}{\longrightarrow} \mathbb{V} \rightarrow \mathbf{V}_{i}$ factors as $\mathbb{H} \times \mathbb{V} \rightarrow \mathbb{H} \times \mathbf{V}_{j} \rightarrow \mathbf{V}_{i}$. Let us denote by $p_{j, i}$ the projection $\mathbf{V}_{j} \rightarrow \mathbf{V}_{i}$ and by act ${ }_{j, i}$ the map $\mathbb{H} \times \mathbf{V}_{j} \rightarrow \mathbf{V}_{i}$.

By the definition of the action, there exists another index $k$ such that the map $\mathbb{H} \times \mathbb{V} \stackrel{\text { act }}{\longrightarrow}$ $\mathbb{V} \rightarrow \mathbf{V}_{j}$ factors as

$$
\mathbb{H} \times \mathbb{V} \rightarrow \mathbb{H} \times \mathbf{V}_{k} \stackrel{\operatorname{act}_{k_{j} j}}{\longrightarrow} \mathbf{V}_{j}
$$

and such that the diagram

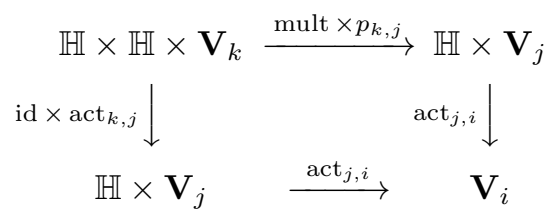

is commutative, where $p_{k, j}$ denotes the projection $\mathbf{V}_{k} \rightarrow \mathbf{V}_{j}$.

Let $\mathbf{W}^{\prime} \subset \mathbf{V}_{j}$ be the kernel of the map $\mathbb{H} \times \mathbf{V}_{j} \stackrel{\text { act }_{j, i}}{\longrightarrow} \mathbf{V}_{i}$, and let $\mathbf{W}^{\prime \prime} \subset \mathbf{V}_{j}$ be the image of $\mathbb{H} \times \mathbf{V}_{k} \stackrel{\text { act }_{k, j}{ }^{j}}{\longrightarrow} \mathbf{V}_{j}$. The above kernel and image are well-defined due to the $(*)$ assumption on $\mathbb{H}$, cf. Sect. 1.10.

Set $\mathbf{V}_{i}^{\prime}$ to be the image of $\mathbf{W}^{\prime \prime}$ in $\mathbf{V}_{j} / \mathbf{W}^{\prime}$, and let act ${ }^{\prime}$ denote the map $\mathbb{H} \times \mathbf{V}_{k} \rightarrow \mathbf{V}_{i}^{\prime}$. We claim that there exists a unique map $\mathbb{H} \times \mathbf{V}_{i}^{\prime} \rightarrow \mathbf{V}_{i}^{\prime}$, which makes the diagram

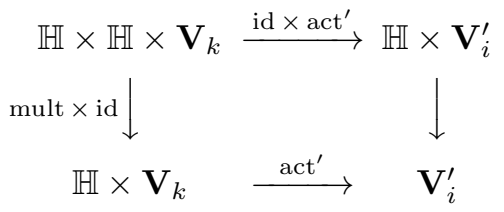

commute. The commutativity of the diagram implies that the action $\mathbb{H} \times \mathbf{V}_{i}^{\prime} \rightarrow \mathbf{V}_{i}^{\prime}$ is unital and associative.

To construct the sought-for map $\mathbb{H} \times \mathbf{V}_{i}^{\prime} \rightarrow \mathbf{V}_{i}^{\prime}$, let us write $\mathbb{H}=$ "lim" $\mathbf{X}_{n}$ with weakly surjective maps. Let $n_{0}$ be an index such that the maps act $t_{j, i}$ and $\operatorname{act}_{j, k}$ are defined on the level of $\mathbf{X}_{n_{0}}$ (we will denote them act $n_{j, i}^{n_{0}}$ and act $_{k, j}^{n_{0}}$, respectively).

Let $n_{1} \geq n_{0}$ be an index such that the multiplication on $\mathbb{H}$ gives rise to a map $\operatorname{mult}_{n_{1}, n_{1}}^{n_{0}}$ : $\mathbf{X}_{n_{1}} \times \mathbf{X}_{n_{1}} \rightarrow \mathbf{X}_{n_{0}}$, and let $n_{2} \geq n_{1}$ be another index, such that we have a multiplication mult $n_{n_{2}, n_{2}}^{n_{1}}: \mathbf{X}_{n_{2}} \times \mathbf{X}_{n_{2}} \rightarrow \mathbf{X}_{n_{1}}$, satisfying an obvious associativity with respect to mult $n_{n_{1}, n_{1}}^{n_{0}}$. For $m=1,2$ let us denote by act $_{j, i}^{n_{m}}$, act ${ }_{k, j}^{n_{m}}$ the maps obtained by composing act ${ }_{j, i}^{n_{0}}$ and act $n_{k, j}^{n_{0}}$, respectively, with $\mathbf{X}_{m} \rightarrow \mathbf{X}_{0}$.

We will construct a map $\mathbf{X}_{n_{2}} \times \mathbf{V}_{i}^{\prime} \rightarrow \mathbf{V}_{i}^{\prime}$, which amounts to a map $\mathbf{X}_{n_{2}}^{\text {top }} \times \mathbf{V}_{i}^{\prime} \rightarrow \mathbf{V}_{i}^{\prime}$. Let $v_{j}$ be an element in $\mathbf{W}^{\prime \prime} \subset \mathbf{V}_{j}$, and $h_{n_{2}} \in \mathbf{X}_{n_{2}}^{\text {top }}$. We claim that there exists an element, denoted 
$v_{j}^{\prime} \in \mathbf{W}^{\prime \prime} \subset \mathbf{V}_{j}$, which is unique modulo $\mathbf{W}^{\prime}$, satisfying

$$
\operatorname{act}_{j, i}^{n_{2}}\left(h_{n_{2}}^{\prime}, v_{j}^{\prime}\right)=\operatorname{act}_{j, i}^{n_{1}}\left(\operatorname{mult}_{n_{2}, n_{2}}^{n_{1}}\left(h_{n_{2}}^{\prime}, h_{n_{2}}\right), v_{j}\right) \in \mathbf{V}_{i},
$$

for any $h_{n_{2}}^{\prime} \in \mathbf{X}_{n_{2}}^{\text {top }}$.

By assumption, every element $v_{j} \in \mathbf{W}^{\prime \prime}$ can be written as $\sum_{a} \operatorname{act}_{k, j}^{n_{2}}\left(h_{n_{2}}^{a}, v_{k}^{a}\right)$ for $h_{n_{2}}^{a} \in \mathbf{X}_{n_{2}}^{\text {top }}$, $v_{k}^{a} \in \mathbf{V}_{k}$. For $h_{n_{2}} \in \mathbf{X}_{n_{2}}^{\text {top }}$ as above we set

$$
v_{j}^{\prime}=\sum_{a} \operatorname{act}_{k, j}^{n_{1}}\left(\operatorname{mult}_{n_{2}, n_{2}}^{n_{1}}\left(h_{n_{2}}, h_{n_{2}}^{a}\right), v_{k}^{a}\right) \in \mathbf{W}^{\prime \prime} \subset \mathbf{V}_{j} .
$$

It is easy to see that $v_{j}^{\prime}$ satisfies (11).

For $\mathbb{H}$ as above we have a natural embedding triv $: \mathbb{V} e c t \rightarrow \operatorname{Rep}(\mathbb{H}, \mathbb{V} e c t)$, corresponding to "trivial" representations.

Corollary 2.6. For $\mathbb{H}$ satisfying (*), the functor triv admits both right and left adjoints.

Note that in Proposition 2.10 a more general statement is established.

Proof. First, from Sect. 1.10 it follows the the functor triv : Vect $\rightarrow \operatorname{Rep}(\mathbb{H}, V e c t)$ admits right and left adjoints, denoted $\Pi \mapsto \Pi^{\mathbb{H}}$ and $\Pi \mapsto \Pi_{\mathbb{H}}$, respectively.

Therefore, using Proposition 2.5] it is enough to show that the functor triv : $\operatorname{Pro}(V e c t) \rightarrow$ $\operatorname{Pro}(\operatorname{Rep}(\mathbb{H}, V e c t))$ has left and right adjoints. But these are simply given by sending $\Pi=$ "lim" $\Pi_{i}$ to $\Pi_{\mathbb{H}} \simeq " \lim ^{\longleftarrow}\left(\Pi_{i}\right)_{\mathbb{H}}$ and $\Pi^{\mathbb{H}} \simeq " \lim "\left(\Pi_{i}\right)^{\mathbb{H}}$, respectively.

As every right adjoint, the functor $\Pi \mapsto \Pi^{\mathbb{H}}$ is left-exact, and similarly, the functor $\Pi \mapsto \Pi_{\mathbb{H}}$ is right-exact.

Lemma 2.7. Assume that $\mathbb{H}$ is the inverse limit of a weakly surjective family of $\mathbf{H}_{i}$, where each $\mathbf{H}_{i}$ is a group-object in $\mathbf{S e t}$ isomorphic to a direct limit of $\mathbf{H}_{i, j}$, with each $\mathbf{H}_{i, j}$ being a group-object of $\operatorname{Pro}\left(\right.$ Set $\left._{0}\right)$. Then the functor of coinvariants $\operatorname{Rep}(\mathbb{H}, \mathbb{V}$ ect $) \rightarrow \mathbb{V e c t}$ is exact.

Proof. According to Proposition 2.5 and Corollary 2.6] each $\Pi \in \operatorname{Rep}(\mathbb{H}, \mathbb{V} e c t)$ is an inverse limit of $\Pi_{k} \in \operatorname{Rep}(\mathbb{H}, V e c t)$, and $\Pi_{\mathbb{H}} \simeq$ "lim" $\left(\Pi_{k}\right)_{\mathbb{H}}$. Therefore, it suffices to show that the functor of coinvariants is exact on $\operatorname{Rep}(\mathbb{H}, \overleftarrow{V e c t})$. By the definition of the latter, we can replace $\mathbb{H}$ by one of its quotients $\mathbf{H}_{i}$, which we will denote by $\mathbf{H}$.

However, the fact that functor $\Pi \mapsto \Pi_{\mathbf{H}}$ is exact on the category $\operatorname{Rep}(\mathbf{H}, V e c t)$ is well-known. Indeed, if $\mathbf{H}=" \lim \mathbf{H}_{j}, \mathbf{H}_{j} \in \operatorname{Pro}\left(\right.$ Set $\left._{0}\right)$,

$$
\Pi_{\mathbf{H}} \simeq \underset{j}{\lim } \Pi_{\mathbf{H}_{j}}
$$

but the functor limInd is exact on Vect, and the functor $\Pi \rightarrow \Pi_{\mathbf{H}_{j}}$ is exact on $\operatorname{Rep}\left(\mathbf{H}_{j}, V e c t\right)$, since $\mathbf{H}_{j}^{\text {top }}$ is a compact group.

2.8. Consider now the category Set with its pseudo-action on $\mathbb{V e c t}$. The main object of study of this paper is the category of representations $\operatorname{Rep}(\mathbb{G}, \mathbb{V} e c t)$ of a group-object $\mathbb{G} \in \mathbb{S e t}$ in $\mathbb{V} e c t$. For brevity, we will denote the category by $\operatorname{Rep}(\mathbb{G})$, when no confusion is likely to occur.

Lemma 2.9. The functor limProj $: \operatorname{Pro}(\operatorname{Rep}(\mathbb{G})) \rightarrow \operatorname{Rep}(\mathbb{G})$ is defined on the entire category and is exact. 
Proof. Recall (cf. Lemma 1.5 with Ind replaced by Pro) that the category $\mathbb{V e c t}$ is closed under projective limits. If $\Pi_{i}=\left(\mathbb{V}_{i}, \rho_{i}\right)$ is an inverse system of objects of $\operatorname{Rep}(\mathbb{G})$, we define $\mathbb{V} \in \mathbb{V}$ ect as $\lim \mathbb{V}_{i}$. It is easy to see from the definitions that there exists an action $\rho: \mathbb{G} \times \mathbb{V} \rightarrow \mathbb{V}$, such that $(\mathbb{V}, \rho)$ represents the projective limit $\lim \Pi_{i}$. The exactness follows from the fact that the functor limProj : Pro $(\mathbb{V e c t}) \rightarrow$ Vect is exact.

We will say that $\mathbb{H} \in \mathbb{S e t}$ satisfies condition $(* *)$ if, as an object of $\operatorname{Ind}(\operatorname{Pro}(\mathbf{S} e t)), \mathbb{H}$ can be represented as "lim" $\mathbb{X}_{k}$, with $\mathbb{X}_{k} \in \operatorname{Pro}(\mathbf{S} e t)$ being weakly strict.

As before, we have an obvious functor triv : $\mathbb{V} e c t \rightarrow \operatorname{Rep}(\mathbb{H})$ corresponding to "trivial" representations.

Proposition 2.10. The functor triv : Vect $\rightarrow \operatorname{Rep}(\mathbb{H})$ admits a left adjoint, and when $\mathbb{H}$ satisfies (**), also a right adjoint.

Proof. Let us first construct the left adjoint of triv. Consider the covariant functor on the category $V$ ect that sends a vector space $\mathbf{V}$ to $\operatorname{Hom}_{\operatorname{Rep}(\mathbb{H})}(\Pi, \operatorname{triv}(\mathbf{V}))$. This functor is a subfunctor of $\mathbf{V} \mapsto \operatorname{Hom}_{\mathbb{V e c t}}(\Pi, \mathbf{V})$. Hence, by Proposition 1.4 it is pro-representable.

Let us denote the resulting object of $\mathbb{V e c t}$ by $\Pi_{\mathbb{H}}$. It is strightforward to check that for $\mathbb{V} \in \mathbb{V} e c t$, we have a functorial isomorphism $\operatorname{Hom}_{\operatorname{Rep}(\mathbb{H})}(\Pi, \operatorname{triv}(\mathbb{V})) \simeq \operatorname{Hom}_{\mathbb{V e c t}}\left(\Pi_{\mathbb{H}}, \mathbb{V}\right)$.

Now let us construct the right adjoint to triv. Let us write $\mathbb{H} \in \mathbb{S e t}$ as $" \underline{l i m} " \mathbb{X}_{k}$, where $\mathbb{X}_{k} \in \operatorname{Pro}(\mathbf{S} e t)$ are weakly strict.

For a weakly strict object $\mathbb{X} \in \operatorname{Pro}(\mathbf{S} e t), \mathbb{V}, \mathbb{U} \in \mathbb{V} e c t$, and an action map $\phi: \mathbb{X} \times \mathbb{V} \rightarrow \mathbb{U}$, consider the kernel of $\phi$ as a functor on $\mathbb{V} e c t$ :

$$
\operatorname{ker}(\phi)(\mathbb{W})=\{\psi: \mathbb{W} \rightarrow \mathbb{V} \mid \phi \circ \psi=0\} .
$$

We claim that this functor is representable. If this is so, it is easy to see that the sought-for right adjoint of triv is representable by

$$
(\mathbb{V}, \rho)^{\mathbb{H}}=\underset{k}{\lim } \operatorname{ker}\left(p-\text { act }: \mathbb{X}_{k} \times \mathbb{V} \rightarrow \mathbb{V}\right)
$$

where $\lim$ is taken in the category $\mathbb{V} e c t$, and $p$ is the obvious projection map $\mathbb{X}_{k} \times \mathbb{V} \rightarrow \mathbb{V}$.

To show the representability, we can assume that $\mathbb{U}=\mathbf{U} \in$ Vect. Indeed, if $\mathbb{U}=$ "lim" $\mathbf{U}_{i}$, then $\operatorname{Ker}(\phi)=\underset{i}{\lim } \operatorname{ker}\left(\mathbb{X} \times \mathbb{V} \rightarrow \mathbf{U}_{i}\right)$. In the latter case, we can assume that $\mathbb{V}=" \overleftarrow{l i m} " \mathbf{V}_{j}$, and we have a compatible system of maps $\phi_{j}: \mathbb{X} \times \mathbf{V}_{j} \rightarrow \mathbf{U}$. By Sect. 1.10 $\operatorname{ker}\left(\phi_{j}\right) \subset \mathbf{V}_{j}$ is well-defined, and it is easy to see that "lim" $\operatorname{ker}\left(\phi_{j}\right) \in \mathbb{V}$ ect represents $\operatorname{ker}(\phi)$.

The main source of examples of such $\mathbb{G}$, i.e., of group-objects in $\mathbb{S e t}$, is provided by considering sets of points of algebraic groups with values in a two-dimensional local field.

2.11. Let $\mathbf{K}$ be a local field, with the corresponding local ring $\mathcal{O}_{\mathbf{K}}$. We will denote by $\pi$ a uniformizer of $\mathbf{K}$. Set $\mathbf{F}=\mathbf{K}((t)), \mathcal{O}_{\mathbf{F}}=\mathbf{K}[[t]]$.

Let $S c h^{f t}$ denote the category of separated schemes of finite type over $\mathbf{K}$. If $S$ is an object of $S c h^{f t}$, we will denote by $S(\mathbf{K})$ the corresponding set of $\mathbf{K}$-points. It is well-known that $S(\mathbf{K})$ carries a natural locally compact totally disconnected topology; therefore, as a topological space, $S(\mathbf{K}) \simeq \mathbf{S}^{\text {top }}$ for a canonically defined locally compact object $\mathbf{S} \in \mathbf{S} e t$. 
Hence, we obtain a functor $S \mapsto \mathbf{S}: S c h^{f t} \rightarrow \mathbf{S e t}$, and also the functors $\operatorname{Pro}\left(S c h^{f t}\right) \rightarrow$ $\operatorname{Pro}(\mathbf{S e t})$, and $\operatorname{Ind}\left(\operatorname{Pro}\left(S c h^{f t}\right)\right) \rightarrow$ Set.

In particular, any affine scheme (not necessarily of finite type) over $\mathbf{K}$ defines an object of $\operatorname{Pro}\left(S c h^{f t}\right)$, and hence, an object of $\operatorname{Pro}(\mathbf{S e t})$. In addition, for any scheme of finite type $S$, the corresponding scheme of $\operatorname{arcs} S[[t]]$ is naturally an object of $\operatorname{Pro}\left(S c h^{f t}\right)$ :

$$
S[[t]] \simeq " \lim _{\longleftarrow} S[t] / t^{i}
$$

We will denote the corresponding object of $\operatorname{Pro}(\mathbf{S} e t)$ by $\mathbf{S}[[t]]$.

If $S$ is smooth, the maps in this family defining $S[[t]]$ are fibrations into affine spaces; therefore the corresponding maps $\mathbf{S}[t] / t^{j} \rightarrow \mathbf{S}[t] / t^{i}$ are weakly surjective. Hence, if $S$ is smooth, the object $\mathbf{S}[[t]] \in \operatorname{Pro}(\mathbf{S} e t)$ is weakly strict.

For a scheme $S^{\prime}$ over $\mathbf{F}$, we define its "restriction of scalars" from $\mathbf{F}$ to $\mathbf{K}$ as a functor on the category of schemes over $\mathbf{K}$ by $S \mapsto \operatorname{Hom}_{\mathbf{F}}\left(S \underset{\mathbf{K}}{\otimes} \mathbf{F}, S^{\prime}\right)$. If $S^{\prime}$ is of finite type and affine, then by embedding it into an affine space one shows that the above functor is ind-representable by an ind-scheme, which is a direct limit of affine schemes under closed embeddings. By taking $S^{\prime}=S \underset{\mathbf{K}}{\otimes} \mathbf{F}$ for $S$ an affine scheme of finite type over $\mathbf{K}$, we obtain an object of $\operatorname{Ind}\left(\operatorname{Pro}\left(S c h^{f t}\right)\right)$ that will be denoted by $S((t))$. The resulting object of $\mathbb{S e t}$ will be denoted by $\mathbf{S}((t))$ or $\mathbb{S}$.

By applying the functor of iterated inductive and projective limits $\mathbb{S e t} \rightarrow$ Set, we obtain from $\mathbb{S}$ (resp., $\mathbf{S}[[t]]$ ) the set, which is tautologically identified with the set $S(\mathbf{F})$ of $\mathbf{F}$-points of $S$ (resp., $S\left(\mathcal{O}_{\mathbf{F}}\right)$-the set of $\mathcal{O}_{\mathbf{F}}$-points of $S$ ).

2.12. If $G$ is a smooth linear algebraic group over $\mathbf{K}$, by applying the functor $G \mapsto \mathbf{G}$ we obtain the corresponding group-object in $\mathbf{S e t}$. In particular, we can consider the category of representations $\operatorname{Rep}(\mathbf{G}, V e c t)$, which is tautologically equivalent to the category of smooth representations of the locally compact group $G(\mathbf{K})$.

For a non-negative integer $i$, let us denote by $G^{i}$ the congruence subgroup of $G[[t]]$, i.e., the kernel of $G[[t]] \rightarrow G[[t]] / t^{i}$; in particular, $G^{0}=G[[t]]$. Let $\mathbf{G}^{i}$ be the corresponding object of $\operatorname{Pro}(\mathbf{S} e t)$. A subgroup $\mathbb{H}$ of $\mathbf{G}[[t]]$ will be called thick if it contains $\mathbf{G}^{i}$ for some $i$ and equals the preimage of a closed subgroup of $\mathbf{G}[[t]] / \mathbf{G}^{i}$ (we are slightly abusing the terminology by identifying $\mathbf{G}[[t]] / \mathbf{G}^{i}$ with the corresponding locally compact group).

For a thick $\mathbb{H} \subset \mathbf{G}[[t]]$ we can consider the corresponding categories $\operatorname{Rep}(\mathbb{H}, V e c t)$ and $\operatorname{Rep}(\mathbb{H}, \mathbb{V} e c t)$. As was remarked above, $\mathbf{G}[[t]] \in \operatorname{Pro}(\mathbf{S} e t)$ is weakly strict, and so are the groups $\mathbf{G}^{i}$. From this it is easy to see that any thick subgroup $\mathbb{H} \subset \mathbf{G}[[t]]$ satisfies condition $(*)$ of Sect. 2.4.

Finally, for an algebraic group $G$ as above, we can consider $\mathbb{G}$ (sometimes also denoted $\mathbf{G}((t)))$, which is a group-object in $\mathbb{S e t}$ and the corresponding category Rep( $\mathbb{G}, \mathbb{V} e c t)$, which we will denote for brevity by $\operatorname{Rep}(\mathbb{G})$.

It is well-known that the ind-scheme $G((t))$ can be represented as a direct limit under closed embeddings of subschemes, each of which is stable under (both left and right) multiplication by $G[[t]]$, and is a principal $G[[t]]$-bundle over a scheme of finite type. (In fact, the above family of subschemes is obtained by taking the preimages of finite-dimensional subschemes of the affine Grassmannian of $G$, i.e., $\operatorname{Gr}_{G}=G((t)) / G[[t]]$.) This implies, in particular, that $\mathbb{G}$ satisfies condition (**), cf. Sect. 2.8.

Let us denote by $\mathbb{V e c t}{ }^{G(\mathbf{F})}$ the category consisting of objects of $\mathbb{V e c t}$ with an action of the abstract group $G(\mathbf{F})$.

Lemma 2.13. The natural forgetful functor $\operatorname{Rep}(\mathbb{G}) \rightarrow \mathbb{V} e c t^{G(\mathbf{F})}$ is fully faithful. 
Proof. We have to show that if $\left(\mathbb{V}_{1}, \rho_{1}\right)$ and $\left(\mathbb{V}_{2}, \rho_{2}\right)$ are two objects of $\operatorname{Rep}(\mathbb{G})$, and $\mathbb{V}_{1} \rightarrow \mathbb{V}_{2}$ is a map preserving the action of $G(\mathbf{F})$, then it is compatible with the $\mathbb{G}$-action.

This can be shown in the following general set-up: Let $\mathbf{V}_{1}, \mathbf{V}_{2}, \mathbf{W}_{1}, \mathbf{W}_{2}$ be vector spaces, and let $\mathbb{X}$ be an object of $\operatorname{Pro}(\mathbf{S} e t)$ endowed with action maps $\mathbb{X} \times \mathbf{V}_{k} \rightarrow \mathbf{W}_{k}, k=1,2$. Let $\mathbf{V}_{1} \rightarrow \mathbf{V}_{2}, \mathbf{W}_{1} \rightarrow \mathbf{W}_{2}$ be maps, such that the square

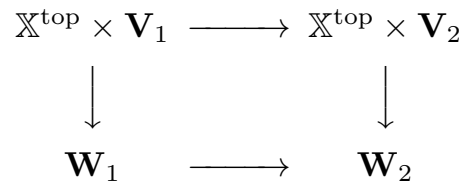

commutes, where $\mathbb{X}^{\text {top }}$ is the topological space obtained from the corresponding object of $\operatorname{Pro}\left(T o p^{H l c t d}\right)$ by taking the projective limit.

Assume now that $\mathbb{X}$ can be presented as "lim" $\mathbf{X}_{i}$, where the maps $\mathbf{X}_{j} \rightarrow \mathbf{X}_{i}$ are such that the corresponding maps $\mathbf{X}_{j}^{\text {top }} \rightarrow \mathbf{X}_{i}^{\text {top }}$ are surjective. Then it is easy to see that the square

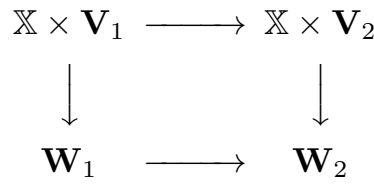

commutes as well.

The above assumption is satisfied in our situation for $\mathbb{X}$ being the object of $\operatorname{Pro}(\mathbf{S} e t)$ corresponding to a subscheme of $G((t))$, obtained as a preimage of a closed subscheme in $G((t)) / G[[t]]$. The required surjectivity follows from the fact that the groups $G^{i}$ for $i>0$ are pro-unipotent.

2.14. Central extensions. Suppose now that $\widehat{G}$ is a group-indscheme, which is a central extension of $G((t))$ by the multiplicative group $G_{m}$, i.e.,

$$
1 \rightarrow G_{m} \rightarrow \widehat{G} \rightarrow G((t)) \rightarrow 1 .
$$

In other words, $\widehat{G}$ is a group-object in the category of ind-schemes, such that if $G((t))=$ "lim" $X_{k}$, and $X_{k}=" \stackrel{l i m}{\longleftarrow} X_{k, l}$ with $X_{k, l} \in S c h^{f t}$, then each $\widehat{G} \underset{G((t))}{\times} X_{k}$ is a total space of a $G_{m}$-torsor over $X_{k}$, and this torsor is pulled back from $X_{k, l}$ for some index $l$. In what follows we will assume that we have a splitting $G[[t]] \rightarrow \widehat{G}$.

We will denote by $\widehat{\mathbb{G}}$ the corresponding group-object in Set, which is an extension of $\mathbb{G}$ by $\mathbf{G}_{m}$.

Let $c$ be a character $G_{m}(\mathbf{K}) \rightarrow \mathbb{C}^{*}$. We will denote by $\operatorname{Rep}_{c}(\widehat{\mathbb{G}})$ the category of representations of $\widehat{\mathbb{G}}$ with central character $c$. In other words, the objects of this category are pairs $\Pi=(\mathbb{V}, \rho)$, where $\mathbb{V} \in \mathbb{V} e c t$, and $\rho$ is an action map $\widehat{\mathbb{G}} \times \mathbb{V} \rightarrow \mathbb{V}$, satisfying the associativity and the unit axioms as above, and such that the composite action

$$
\mathbf{G}_{m} \times \mathbb{V} \rightarrow \widehat{\mathbb{G}} \times \mathbb{V} \rightarrow \mathbb{V}
$$

(where $\mathbf{G}_{m}$ is viewed as an object of $\mathbf{S e t} \subset \mathbb{S e t}$ ) corresponds to the above character.

2.15. We propose the category $\operatorname{Rep}(\mathbb{G})=\operatorname{Rep}(\mathbb{G}, \mathbb{V} e c t)$ as a framework for the study of representations of the group $G(\mathbf{F})$. Let us explain why introducing pro-objects of $V e c t$ appears to be necessary. For the remainder of this section, let us assume that $G$ is semi-simple, simplyconnected and split. 
The first question to ask is whether the category $\operatorname{Rep}(\mathbb{G})$ contains any objects $\Pi=(\mathbf{V}, \rho)$, where $\mathbf{V}$ belongs to $V$ ect. The answer is that such representations are necessarily trivial (i.e., they lie in the image of the functor $V e c t \rightarrow \mathbb{V e c t} \stackrel{\text { triv }}{\rightarrow} \operatorname{Rep}(\mathbb{G})$ ), for the same reason as why $\mathfrak{p}$-adic groups usually have no finite-dimensional representations.

Indeed, suppose that $(\mathbf{V}, \rho)$ is such a representation. By Lemma 2.13 it is sufficient to prove that the corresponding representation of the abstract group $G(\mathbf{F})$ on $\mathbf{V}$ is trivial.

Consider the kernel $K$ of the action $G(\mathbf{F}) \times \mathbf{V} \rightarrow \mathbf{V}$. This is a normal subgroup, and by definition, there exists an $i$ such that $K \supset G^{i}(\mathbf{K})$. But then we claim that $K$ must coincide with $G(\mathbf{F})$. Let $N$ be the maximal unipotent subgroup of $G$, and let $N^{i}(\mathbf{K}):=N(\mathbf{F}) \cap G^{i}(\mathbf{K})$ be the corresponding congruence subgroup. Then $N^{i}(\mathbf{K}) \subset K$, but using the torus action and the normality of $K$, we obtain that the entire $N(\mathbf{F})$ is contained in $K$. Again, by normality, we obtain that all unipotent elements in $G(\mathbf{F})$ are contained in $K$. However, it is known that for a split simply-connected group, its set of field-valued points is generated by the subset of unipotent elements.

Another sense in which one may seek an alternative definition of $G(\mathbf{F})$-representations is to consider the pseudo-action of $\mathbb{S e t}$ on $\operatorname{Ind}(V e c t)=\operatorname{Ind}\left(\operatorname{Ind}\left(V e c t_{0}\right)\right)$. We claim that (under the same assumption on $G$ ) all objects of $\operatorname{Rep}(\mathbb{G}, \operatorname{Ind}(V e c t))$ are again trivial.

Proof. As before, we have a fully faithful functor $\operatorname{Rep}(\mathbb{G}, \operatorname{Ind}(V e c t)) \rightarrow \operatorname{Ind}(V e c t)^{G(\mathbf{F})}$, and it suffices to show that for any object $(\mathbf{V}, \rho), \mathbf{V} \in \operatorname{Ind}(V e c t)$, the action of the maximal unipotent group $N(\mathbf{F})$ on $\mathbf{V}$ is trivial. Obviously, we can replace $G$ by an $S L_{2}$ corresponding to some simple root; let $B \subset G$ be the corresponding Borel subgroup, i.e., $N \simeq G_{a}$, and $B:=G_{a} \ltimes G_{m}$, where $G_{m}$ acts on $G_{a}$ by the square of the standard character.

Our $\mathbf{V}$ is a direct limit "lim" $\mathbf{V}_{l}$, with $\mathbf{V}_{l} \in V$ Vect. Fix an index $l$, and it suffices to show that the action map $B(\mathbf{F}) \times \overrightarrow{\mathbf{V}_{l}} \rightarrow \mathbf{V}$ is trivial.

For a (not necessarily positive) integer $i$, let us denote by $N^{i}(\mathbf{K})$ the subgroup of $N(\mathbf{F}) \simeq$ $\mathbf{K}((t))$ equal to $t^{i} \cdot \mathbf{K}[[t]]$. If the action of $N(\mathbf{F})$ on $\mathbf{V}_{l}$ is non-trivial, let $i$ be the minimal integer such that the restriction of this action to $N^{i}(\mathbf{K})$ is trivial. By assumption we have a non-trivial action $\operatorname{map}\left(N^{i-1}(\mathbf{K}) / N^{i}(\mathbf{K}) \simeq \mathbf{K}\right) \times \mathbf{V}_{l} \rightarrow \mathbf{V}$.

Let now $j$ be a sufficiently large integer, so that the corresponding congruence subgroup $\left(G_{m}\right)^{j}(\mathbf{K})$ acts trivially on $\mathbf{V}_{l}$. Take $i^{\prime}=(i-1)-2 j$ and consider now the action of $N^{i^{\prime}}(\mathbf{K})$ on $\mathbf{V}_{l}$. Let $l^{\prime}$ be a sufficiently large index such that the iteration of actions

$$
N^{i^{\prime}}(\mathbf{K}) \times\left(G_{m}\right)^{j}(\mathbf{K}) \times N^{i^{\prime}}(\mathbf{K}) \times\left(G_{m}\right)^{j}(\mathbf{K}) \times \mathbf{V}_{l} \rightarrow \mathbf{V}_{l^{\prime}}
$$

is well-defined. We will show that the action $N^{i-1}(\mathbf{K}) / N^{i}(\mathbf{K}) \times \mathbf{V}_{l} \rightarrow \mathbf{V}_{l^{\prime}}$ is necessarily trivial, which would be a contradiction. For that, it suffices to show that it is trivial on every element $v \in \mathbf{V}_{l}$. For every such $v$ there exists an integer $k$ such that the action of $t^{i^{\prime}} \cdot \pi^{k} \cdot \mathcal{O}_{\mathbf{K}}[[t]] \subset N^{i^{\prime}}(\mathbf{K})$ on $v$ is trivial. Hence, for $g \in\left(G_{m}\right)^{j}(\mathbf{K})$ and $n \in t^{i^{\prime}} \cdot \pi^{k} \cdot \mathcal{O}_{\mathbf{K}}[[t]]$

$$
\left(n \cdot g \cdot n^{-1} \cdot g^{-1}\right) \cdot v=v \in \mathbf{V}_{l^{\prime}} .
$$

However, since $\left(G_{m}\right)^{j}(\mathbf{K})=1+t^{j} \cdot \mathbf{K}[[t]]$, the subset of $N(\mathbf{K})$ consisting of elements of the form $\left(n \cdot g \cdot n^{-1} \cdot g^{-1}\right)$, with $g$ and $n$ as above, equals the entire $t^{i} \cdot \mathbf{K}[[t]]$, in particular, it projects surjectively onto $N^{i-1}(\mathbf{K}) / N^{i}(\mathbf{K})$. Therefore, for $n^{\prime} \in N^{i-1}(\mathbf{K})$, we have $n^{\prime} \cdot v=v \in \mathbf{V}_{l^{\prime}}$, which is what we had to show. 


\section{THE INDUCTION FUNCTOR}

3.1. Let $G$ be a split reductive group over $\mathbf{K}$, and let $\mathbb{H}$ be a thick subgroup of $\mathbf{G}[[t]]$. We have an obvious restriction functor $r_{\mathbb{H}}^{\mathbb{G}}: \operatorname{Rep}(\mathbb{G}) \rightarrow \operatorname{Rep}(\mathbb{H}, \mathbb{V} e c t)$.

Our goal in this section is define the functors $\widetilde{i}_{\mathbb{H}}^{\mathbb{G}}, i_{\mathbb{H}}^{\mathbb{G}}: \operatorname{Rep}(\mathbb{H}, \mathbb{V e c t}) \rightarrow \operatorname{Rep}(\mathbb{G})$, such that $\widetilde{i}_{\mathbb{H}}^{\mathbb{G}}$ will be the right adjoint of $r_{\mathbb{H}}^{\mathbb{G}}$.

We will have an injective functorial map $i_{\mathbb{H}}^{\mathbb{G}}(\Pi) \rightarrow \widetilde{\mathbb{i}_{\mathbb{H}}}(\Pi)$, and there is a certain analogy between the functors $\widetilde{i}_{\mathbb{H}}^{\mathbb{G}}$ and $i_{\mathbb{H}}^{\mathbb{G}}$ and the functors of induction and compact induction in the theory of $\mathfrak{p}$-adic groups. When $\mathbb{H}$ corresponds to a parahoric subgroup of $G[[t]]$, we will have an isomorphism $i_{\mathbb{H}}^{\mathbb{G}} \simeq \widetilde{i_{\mathbb{H}}^{\mathbb{G}}}$.

The construction of the functor $i_{\mathbb{H}}^{\mathbb{G}}$ makes sense for any algebraic group $G$, but the construction of the functor $\widetilde{i}_{\mathbb{H}}^{\mathbb{G}}$ given below uses the fact that $G$ is reductive. However, we expect that the right adjoint to $r_{\mathbb{H}}^{\mathbb{G}}$ exists for any $G$.

3.2. To an object $\mathbf{X} \in \operatorname{Pro}\left(\operatorname{Set}_{0}\right)$ we can attach the vector space of locally constant $\mathbb{C}$-valued functions, denoted Funct ${ }^{l c}(\mathbf{X})$. Namely, if $\mathbf{X}=$ "lim" $X_{i}$,

$$
\operatorname{Funct}^{l c}(\mathbf{X})=\underset{\lim }{\longrightarrow} \operatorname{Funct}\left(X_{i}\right)
$$

where the direct system is taken with respect to the pull-back maps between the spaces of functions. Of course, Funct ${ }^{l c}(\mathbf{X})$ identifies with the space of locally constant functions on the topological space $\mathbf{X}^{\text {top }}$.

For any $\mathbf{X} \in \mathbf{S e t}$ we define $\mathbb{F} u n c t^{l c}(\mathbf{X}) \in \mathbb{V e c t}$ by setting for $" \underline{l i m} " \mathbf{X}_{j}, \mathbf{X}_{j} \in \operatorname{Pro}\left(\right.$ Set $\left._{0}\right)$

$$
\mathbb{F} \text { unct }{ }^{l c}(\mathbf{X})=" l i m " \operatorname{Funct}^{l c}\left(\mathbf{X}_{j}\right)
$$

with respect to the restriction maps. We define the space Funct ${ }^{l c}(\mathbf{X}) \in V e c t$ of locally constant functions on $\mathbf{X}$ as $\lim \operatorname{Proj}\left(\mathbb{F} u n c t^{l c}(\mathbf{X})\right)$.

If $\mathbf{X} \in \mathbf{S} e t$ is locally compact (cf. Sect. 1.6), we can introduce the vector space Funct ${ }_{c}^{l c}(\mathbf{X})$, which can be called the space of locally constant functions with compact support. One way to introduce it is as the space of locally-constant compactly supported functions on $\mathbf{X}^{\text {top }}$. Equivalently, if $\mathbf{X}$ is represented as a direct limit as in Sect. [1.6] we have the natural "extension by zero" maps Funct ${ }^{l c}\left(\mathbf{X}_{i}\right) \rightarrow$ Funct $^{l c}\left(\mathbf{X}_{j}\right)$, and we set Funct ${ }_{c}^{l c}(\mathbf{X})=\stackrel{\lim }{\longrightarrow}$ Funct $^{l c}\left(\mathbf{X}_{i}\right)$. Note that we always have an inclusion Funct $_{c}^{l c}(\mathbf{X}) \hookrightarrow$ Funct $^{l c}(\mathbf{X})$.

Let $\mathbf{X}$ be again locally compact, presented as a direct limit as in Sect. 1.6 If $\mathbf{X}^{\prime} \rightarrow \mathbf{X}$ is map between objects of $\mathbf{S e t}$, we define the vector space Funct $t_{c, r e l}^{l c}\left(\mathbf{X}^{\prime}\right)$ as the inductive limit $\stackrel{\lim }{\longrightarrow} \operatorname{Funct}^{l c}\left(\mathbf{X}^{\prime} \underset{\mathbf{X}}{\times} \mathbf{X}_{i}\right)$.

If $\mathbf{X} \rightarrow \mathbf{Y}$ is a map in $\mathbf{S e t}$, we have the pull-back morphism $\operatorname{Funct}^{l c}(\mathbf{Y}) \rightarrow \operatorname{Funct}^{l c}(\mathbf{X})$, and if this is a proper map between locally compact objects, we also have the morphism Funct ${ }_{c}^{l c}(\mathbf{Y}) \rightarrow$ Funct $_{c}^{l c}(\mathbf{X})$.

Suppose now that $\mathbf{Y}^{1}, \mathbf{Y}^{2} \in \mathbf{S}$ et are locally compact, and we have an action $\mathbf{X} \times \mathbf{Y}^{1} \rightarrow \mathbf{Y}^{2}$ (in the sense of the canonical tensor structure on $\mathbf{S e t}$ ), such that the map $\mathbf{X} \times \mathbf{Y}^{1} \rightarrow \mathbf{X} \times \mathbf{Y}^{2}$ is proper. Then we obtain an action map $\mathbf{X} \times \operatorname{Funct}_{c}^{l c}\left(\mathbf{Y}^{2}\right) \rightarrow \operatorname{Funct}_{c}^{l c}\left(\mathbf{Y}^{1}\right)$ (in the sense of the pseudo-action of Set on Vect).

For example, the above properness condition is always satisfied if $\mathbf{X}$ is a group-object acting on $\mathbf{Y}^{1}=\mathbf{Y}^{2}$.

Note that the above action does not always extend onto Funct ${ }^{l c}(\mathbf{Y})$. 
Let now $\mathbf{Y}$ be an object of $\operatorname{Ind}(\mathbf{S} e t)$. We will say that $\mathbf{Y}$ is "tame" if it can be represented as "lim" $\mathbf{Y}_{i}$ such that $\mathbf{Y}_{i} \in \mathbf{S e t}$ are locally compact, and the corresponding maps $\mathbf{Y}_{i} \rightarrow \mathbf{Y}_{j}$ are proper. If $\mathbf{Y}$ is "tame", we can attach to it the object Funct ${ }_{c}^{l c}(\mathbf{Y}) \in \operatorname{Vect}_{\text {as }} \operatorname{Funct}_{c}^{l c}(\mathbf{Y})=$ " $\lim$ Funct $_{c}^{l c}\left(\mathbf{Y}_{i}\right)$, where the maps are again given by restriction.

Suppose now that $\mathbf{Y}^{1}, \mathbf{Y}^{2} \in \operatorname{Ind}(\mathbf{S} e t)$ are both "tame", $\mathbf{Y}_{i}^{j}=$ "lim" $\mathbf{Y}_{i}^{j}$ for $j=1,2$, and let $\mathbb{X} \times \mathbf{Y}^{1} \rightarrow \mathbf{Y}^{2}$ be an action of $\mathbb{X} \in \mathbb{S e t}$ in the sense of the pseudo-action of $\operatorname{Set}$ on $\operatorname{Ind}(\mathbf{S e t})$. That is $\mathbb{X}=$ "lim" $\mathbb{X}_{l}, \mathbb{X}_{l}=$ "lim" $\mathbf{X}_{l, k}$ and the action is given by the maps $\mathbf{X}_{l, k} \times \mathbf{Y}_{i}^{1} \rightarrow \mathbf{Y}_{i^{\prime}}^{2}$ We say that this action is proper if the above presentations can be chosen so that the maps $\mathbf{X}_{l, k} \times \mathbf{Y}_{i}^{1} \rightarrow \mathbf{X}_{l, k} \times \mathbf{Y}_{i^{\prime}}^{2}$ are proper. (This condition is satisfied if $\mathbb{H}$ is a group-object in $\mathbb{S e t}$ acting on $\mathbf{Y}=\mathbf{Y}^{1}=\mathbf{Y}^{2}$.)

If the action $\mathbb{X} \times \mathbf{Y}^{1} \rightarrow \mathbf{Y}^{2}$ is proper we obtain an action map $\mathbb{X} \times$ Funct $_{c}^{l c}\left(\mathbf{Y}_{2}\right) \rightarrow$ Funct $_{c}^{l c}\left(\mathbf{Y}_{1}\right)$ in the sense of the canonical pseudo-action of Set on $\mathbb{V} e c t$.

A little more generally, if $\mathbf{V}$ is a vector space, instead of complex-valued functions, we can consider spaces of functions with values in $\mathbf{V}$, denoted $\mathrm{Funct}^{l c}(\mathbf{X}, \mathbf{V})$ and $\mathrm{Funct}_{c}^{l c}(\mathbf{X}, \mathbf{V})$, respectively.

3.3. Let $i \geq 0$ be such that $\mathbf{G}^{i} \subset \mathbb{H}$. Consider the full subcategory of $\operatorname{Rep}\left(\mathbb{H} / \mathbf{G}^{i}, V e c t\right) \subset$ $\operatorname{Rep}(\mathbb{H}, \mathbb{V} e c t)$; we will first define the restrictions of the functors $i_{\mathbb{H}}^{\mathbb{G}}, i_{\mathbb{H}}^{\mathbb{G}}$ to this subcategory.

Recall that there exists a strict ind-scheme of ind-finite-type $G((t)) / G^{i}$ ("strict" means that it can be presented as a direct limit of schemes with transition maps being closed embedding). Its existence, i.e., the ind-representability of the corresponding functor, follows easily from the corresponding fact for $\mathrm{Gr}_{G}=G((t)) / G[[t]]$ (see, for example, the Appendix to [] $]$ ). As an object of $\operatorname{Ind}\left(S c h^{f t}\right)$ it carries an action of $G((t)) \in \operatorname{Ind}\left(\operatorname{Pro}\left(S c h^{f t}\right)\right)$ "on the left" and a commuting action of $G\left([[t]] / t^{i}\right) \in S c h^{f t}$ "on the right".

Therefore, by applying the functor $S \mapsto \mathbf{S}: S c h^{f t} \rightarrow \mathbf{S} e t$, we obtain a "tame" object, denoted $\mathbb{G} / \mathbf{G}^{i}$ in $\operatorname{Ind}(\mathbf{S} e t)$, which carries the actions of $\mathbb{G}$ and $\mathbf{G}[[t]] / \mathbf{G}^{i}$.

For an object $\Pi=(\mathbf{V}, \rho) \in \operatorname{Rep}\left(\mathbb{H} / \mathbf{G}^{i}, V e c t\right)$, we obtain that Funct ${ }_{c}^{l c}\left(\mathbb{G}((t)) / \mathbf{G}^{i}, \mathbf{V}\right) \in \mathbb{V} e c t$ carries a natural $\mathbb{G}$-action and a commuting $\mathbb{H} / \mathbf{G}^{i}$-action.

The object of $\mathbb{V e c t}$ underlying $i_{\mathbb{H}}^{\mathbb{G}}(\Pi)$ is set to be

$$
\left(\operatorname{Funct}_{c}^{l c}\left(\mathbb{G} / \mathbf{G}^{i}, \mathbf{V}\right) \otimes \mu\left(\mathbb{H} / \mathbf{G}^{i}\right)\right)_{\mathbb{H} / \mathbf{G}^{i}},
$$

where $\mu\left(\mathbb{H} / \mathbf{G}^{i}\right)$ is the 1-dimensional vector space of left-invariant measures on the locally compact group $\left(\mathbb{H} / \mathbf{G}^{i}\right)^{\text {top }}$ (acted on naturally by $\mathbb{H} / \mathbf{G}^{i}$, being a subspace of all measures on $\left.\left(\mathbb{H} / \mathbf{G}^{i}\right)^{\text {top }}\right)$. The $\mathbb{G}$-action on Funct ${ }_{c}^{l c}\left(\mathbb{G} / \mathbf{G}^{i}, \mathbf{V}\right)$ defines on $i_{\mathbb{H}}^{\mathbb{G}}(\Pi)$ a structure of an object of $\operatorname{Rep}(\mathbb{G})$.

This definition of $i_{\mathbb{H}}^{\mathbb{G}}(\Pi)$ can be rewritten as follows. First, let us introduce the object $\mathbb{G} / \mathbb{H} \in \operatorname{Ind}(\mathbf{S} e t)$. Let us write $G((t)) / G[[t]]$ as "lim" $S_{k}, S_{k} \in S c h^{f t}$, and let $S_{k}^{i}$ be the preimage of $S_{k}$ in $G((t)) / G^{i}$. Let $\mathbf{S}_{k}, \mathbf{S}_{k}^{i}$ be the corresponding objects of $\mathbf{S} e t$. By construction $\mathbf{S}_{k}^{i}$ carries an action of the groups $\mathbb{H} \subset \mathbf{G}[[t]] / \mathbf{G}^{i}$, and we claim that the categorical quotient $\mathbf{S}_{k}^{\mathbb{H}}:=\left(\mathbf{S}_{k}^{i}\right) /\left(\mathbb{H} / \mathbf{G}^{i}\right) \in \mathbf{S} e t$ is well-defined and is locally compact. This follows for example from the fact that $S_{k}^{i} \rightarrow S_{k}$ is a fibration locally trivial in the Zariski toplogy. Let $\mathbb{G} / \mathbb{H}=$ "lim" $\mathbf{S}_{k}^{\mathbb{H}}$ be the corresponding object of Ind(Set); this object is "tame" and it evidently does not depend on the way we presented $G((t)) / G[[t]] \in \operatorname{Ind}\left(S c h^{f t}\right)$ as a direct limit. 
For $\Pi=(\mathbf{V}, \rho) \in \operatorname{Rep}\left(\mathbb{H} / \mathbf{G}^{i}, V e c t\right)$, let Funct ${ }_{c, r e l}^{l c}\left(\mathbf{S}_{k}^{i}, \mathbf{V}\right)$ be the space of locally-constant $\mathbf{V}$-valued functions on $\mathbf{S}_{k}^{i}$, whose support is contained in the preimage of a compact subset of $\mathbf{S}_{k}^{\mathbb{H H}}$ (see Sect. 3.2).

We have:

$$
\left(\operatorname{Funct}_{c}^{l c}\left(\mathbf{S}_{k}^{i}, \mathbf{V}\right) \otimes \mu\left(\mathbb{H} / \mathbf{G}^{i}\right)\right)_{\mathbb{H} / \mathbf{G}^{i}} \simeq\left(\text { Funct }_{c, r e l}^{l c}\left(\mathbf{S}_{k}^{i}, \mathbf{V}\right)\right)^{\mathbb{H} / \mathbf{G}^{i}}
$$

where the isomorphism is given by integration along the fibers of $\mathbf{S}_{k}^{i} \rightarrow \mathbf{S}_{k}^{\mathbb{H}}$.

The above isomorphism makes it clear that $i_{\mathbb{H}}^{\mathbb{G}}(\Pi)$, as an object of $\operatorname{Rep}(\mathbb{G})$, is independent of the choice of the congruence subgroup $\mathbf{G}^{i}$ contained in $\mathbb{H}$. In particular, we obtain a welldefined functor $i_{\mathbb{H}}^{\mathbb{G}}: \operatorname{Rep}(\mathbb{H}, V e c t) \rightarrow \operatorname{Rep}(\mathbb{G})$. From (2) we infer that $i_{\mathbb{H}}^{\mathbb{G}}$ is right-exact, and from (3) that it is also left-exact.

Using Proposition 2.5 we extend the above functor $\operatorname{Rep}(\mathbb{H}, V e c t) \rightarrow \operatorname{Rep}(\mathbb{G})$ to a functor $\operatorname{Rep}(\mathbb{H}, \mathbb{V} e c t) \simeq \operatorname{Pro}(\operatorname{Rep}(\mathbb{H}, V e c t)) \rightarrow \operatorname{Pro}(\operatorname{Rep}(\mathbb{G}))$, which is also exact. We extend it further to an exact functor $i_{\mathbb{H}}^{\mathbb{G}}: \operatorname{Rep}(\mathbb{H}, \mathbb{V e c t}) \rightarrow \operatorname{Rep}(\mathbb{G})$, using Lemma 2.9

It is easy to see that our functor $i_{\mathbb{H}}^{\mathbb{G}}$ is isomorphic to the composition of two functors: $\left.i_{\mathbf{G}}^{\mathbb{G}}[t]\right]$ : $\operatorname{Rep}(\mathbf{G}[[t]], \mathbb{V} e c t) \rightarrow \operatorname{Rep}(\mathbb{G})$ and $i_{\mathbb{H}}^{\mathbf{G}}[[t]]: \operatorname{Rep}(\mathbb{H}, \mathbb{V} e c t) \rightarrow \operatorname{Rep}(\mathbf{G}[[t]], \mathbb{V} e c t)$, where the latter functor is defined by a similar induction procedure.

3.4. Let us now define the functor $\widetilde{i_{\mathbb{H}}^{\mathbb{G}}}: \operatorname{Rep}(\mathbb{H}, \mathbb{V} e c t) \rightarrow \operatorname{Rep}(\mathbb{G})$. First, let us assume that $\mathbb{H}$ is such that $\mathbb{G} / \mathbb{H} \in \operatorname{Ind}(\mathbf{S} e t)$ is ind-compact, i.e., is a direct limit of compact objects of $\mathbf{S e t}$. (E.g., this condition is verified for $\mathbf{G}[[t]]$, or more generally for any $\mathbb{H}$ containing $\mathbf{I}$, where $I \subset G[[t]]$ is the Iwahori subgroup. This follows from the fact that the affine flag variety $G((t)) / I$ is ind-proper, i.e., is a direct limit of proper schemes of finite type.)

In this case we set $\widetilde{i}_{\mathbb{H}}^{\mathbb{G}}=i_{\mathbb{H}}^{\mathbb{G}}$.

Proposition 3.5. If $\mathbb{G} / \mathbb{H}$ is ind-compact, the functor $i_{\mathbb{H}}^{\mathbb{G}}$ is the right adjoint to the restriction functor $r_{\mathbb{H}}^{\mathbb{G}}$.

Proof. The proof mimics the proof of the usual adjunction property for $\mathfrak{p}$-adic groups.

Let us first construct the adjunction map $r_{\mathbb{H}}^{\mathbb{G}} \circ i_{\mathbb{H}}^{\mathbb{G}} \rightarrow \mathrm{id}_{\operatorname{Rep}(\mathbb{H}, \text { Vect })}$. By the definition of $i_{\mathbb{H}}^{\mathbb{G}}$, it is enough to construct a morphism $r_{\mathbb{H}}^{\mathbb{G}} \circ i_{\mathbb{H}}^{\mathbb{G}}(\Pi) \rightarrow \Pi$ for an object of $\operatorname{Rep}\left(\mathbb{H} / \mathbf{G}^{i}, V e c t\right)$ for some $i$.

For $\Pi=(\mathbf{V}, \rho) \in \operatorname{Rep}\left(\mathbb{H} / \mathbf{G}^{i}, V e c t\right)$, consider the canonical restriction map

$$
\operatorname{Funct}_{c}^{l c}\left(\mathbb{G} / \mathbf{G}^{i}, \mathbf{V}\right) \otimes \mu\left(\mathbb{H} / \mathbf{G}^{i}\right) \rightarrow \operatorname{Funct}_{c}^{l c}\left(\mathbb{H} / \mathbf{G}^{i}, \mathbf{V}\right) \otimes \mu\left(\mathbb{H} / \mathbf{G}^{i}\right),
$$

which is bi- $\mathbb{H} / \mathbf{G}^{i}$-equivariant by construction.

Since Funct ${ }_{c}^{l c}\left(\mathbb{H} / \mathbf{G}^{i}\right) \otimes \mu\left(\mathbb{H} / \mathbf{G}^{i}\right)$ identifies as a bi-module over $\left(\mathbb{H} / \mathbf{G}^{i}\right)^{\text {top }}$ with the Hecke algebra of (compactly supported, locally constant) measures on this group, we obtain a bi$\mathbb{H} / \mathbf{G}^{i}$-equivariant map Funct $c_{c}^{l c}\left(\mathbb{H} / \mathbf{G}^{i}, \mathbf{V}\right) \otimes \mu\left(\mathbb{H} / \mathbf{G}^{i}\right) \rightarrow \mathbf{V}$. By the $\mathbb{H} / \mathbf{G}^{i}$-equivariance on the right, we thus obtain a map $\left(\text { Funct }_{c}^{l c}\left(\mathbb{G} / \mathbf{G}^{i}, \mathbf{V}\right) \otimes \mu\left(\mathbb{H} / \mathbf{G}^{i}\right)\right)_{\mathbb{H} / \mathbf{G}^{i}} \rightarrow \mathbf{V}$, as required.

Let us now construct the second adjunction map $\left(\mathbb{W}, \rho^{\prime}\right) \rightarrow i_{\mathbb{H}}^{\mathbb{G}} \circ r_{\mathbb{H}}^{\mathbb{G}}\left(\mathbb{W}, \rho^{\prime}\right)$ for $\left(\mathbb{W}, \rho^{\prime}\right) \in$ $\operatorname{Rep}(\mathbb{G})$. Using Proposition [2.5] we can represent $r_{\mathbb{H}}^{\mathbb{G}}(\mathbb{W})$ as an inverse limit of $\mathbf{W}_{i}$, where each $\mathbf{W}_{i}$ is a vector space underlying an object of $\operatorname{Rep}\left(\mathbb{H} / \mathbf{G}^{n_{i}}, V e c t\right)$ for some $n_{i}$. Let $\mathbb{G} / \mathbb{H}=$ "lim" $\mathbf{S}_{k}^{\mathbb{H}}$ be as before, and let $\mathbf{S}_{k}^{n_{i}}$ be the preimage of $\mathbf{S}_{k}^{\mathbb{H}}$ in $\mathbb{G} / \mathbf{G}^{n_{i}}$. 
Then the object of $\mathbb{V} e c t$ underlying $i_{\mathbb{H}}^{\mathbb{G}} \circ r_{\mathbb{H}}^{\mathbb{G}}\left(\mathbb{W}, \rho^{\prime}\right)$ is

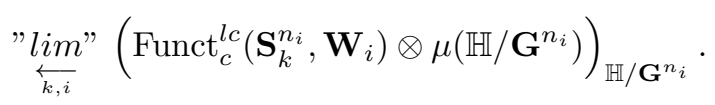

For every fixed $k$ and $i$, let an index $j$ be such that the $\mathbb{G}$-action on $\mathbb{W}$ gives a map act ${ }_{j, i}$ : $\mathbf{S}_{k}^{n_{j}} \times \mathbf{W}_{j} \rightarrow \mathbf{W}_{i}$. By further enlarging $j$, we may assume that this map is compatible with the $\mathbb{H}$-action.

We define a map $\mathbf{W}_{j} \rightarrow\left(\text { Funct }_{c}^{l c}\left(\mathbf{S}_{k}^{n_{i}}, \mathbf{W}_{i}\right) \otimes \mu\left(\mathbb{H} / \mathbf{G}^{n_{i}}\right)\right)_{\mathbb{H} / \mathbf{G}^{n_{i}}}$ as follows. First, the above action map gives rise to a map

$$
\mathbf{W}_{j} \rightarrow\left(\text { Funct }^{l c}\left(\mathbf{S}_{k}^{n_{j}}, \mathbf{W}_{i}\right)\right)^{\mathbb{H} / \mathbf{G}^{n_{j}}} .
$$

Now, from the fact that $\mathbf{S}_{k}^{\mathbb{H}}$ is compact and isomorphism (3), we obtain

$$
\left(\operatorname{Funct}^{l c}\left(\mathbf{S}_{k}^{n_{j}}, \mathbf{W}_{i}\right)\right)^{\mathbb{H} / \mathbf{G}^{n_{j}}} \simeq\left(\operatorname{Funct}_{c}^{l c}\left(\mathbf{S}_{k}^{n_{i}}, \mathbf{W}_{i}\right) \otimes \mu\left(\mathbb{H} / \mathbf{G}^{n_{i}}\right)\right)_{\mathbb{H} / \mathbf{G}^{n_{i}}} .
$$

By composing, we obtain the required morphism.

It is easy to check that the constructed map from $\mathbb{W}$ to the object of $\mathbb{V} e c t$ underlying $i_{\mathbb{H}}^{\mathbb{G}} \circ r_{\mathbb{H}}^{\mathbb{G}}(\mathbb{W})$ respects the $\mathbb{G}$-action. It is equally straightforward to see that the two adjunction maps indeed give rise to the adjointness of functors.

Thus, to define the functor $\widetilde{i_{\mathbb{H}}^{\mathbb{G}}}$ in general, it suffices to define the functor $\widetilde{i}_{\mathbb{H}}^{\mathbf{G}}[[t]]$, which is the right adjoint to the restriction functor $r_{\mathbb{H}}^{\mathbf{G}[[t]]}: \operatorname{Rep}(\mathbf{G}[[t]], \mathbb{V} e c t) \rightarrow \operatorname{Rep}(\mathbb{H}, \mathbb{V} e c t)$.

Let $\mathbf{G}^{i}$ be a congruence subgroup contained in $\mathbb{H}$. We define the functor

$$
\widetilde{i}_{\mathbb{H}}^{\mathbf{G}[[t]]}: \operatorname{Rep}\left(\mathbb{H} / \mathbf{G}^{i}, \text { Vect }\right) \rightarrow \operatorname{Rep}\left(\mathbf{G}[[t]] / \mathbf{G}^{i}, V e c t\right)
$$

to equal the corresponding functor defined for locally compact groups.

Explicitly, for $\Pi=(\mathbf{V}, \rho) \in \operatorname{Rep}\left(\mathbb{H} / \mathbf{G}^{i}, V e c t\right)$,

$$
\widetilde{i}_{\mathbb{H}}^{\mathbf{G}}[[t]] \simeq\left(\text { Funct }^{s m}\left(\mathbf{G}[[t]] / \mathbf{G}^{i}, \mathbf{V}\right)\right)^{\mathbb{H} / \mathbf{G}^{i}}
$$

where Funct ${ }^{s m}\left(\mathbf{G}[[t]] / \mathbf{G}^{i}\right)$ is the space of functions on $\mathbf{G}[[t]] / \mathbf{G}^{i}$, smooth with respect to the action of this group by left translations.

Note that for $\Pi=(\mathbf{V}, \rho) \in \operatorname{Rep}\left(\mathbb{H} / \mathbf{G}^{i}, V e c t\right)$ as above, the object of $V e c t$ underlying $\widetilde{i}_{\mathbb{H}}^{\mathbb{G}} \circ$

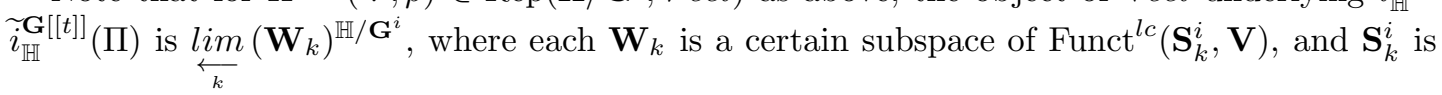
as in (2).

The above functor $\operatorname{Rep}\left(\mathbb{H} / \mathbf{G}^{i}, V e c t\right) \rightarrow \operatorname{Rep}\left(\mathbf{G}[[t]] / \mathbf{G}^{i}, V e c t\right)$ extends to a functor $\widetilde{i}_{\mathbb{H}}^{\mathbf{G}}[[t]]:$ $\operatorname{Rep}(\mathbb{H}, V e c t) \rightarrow \operatorname{Rep}(\mathbf{G}[[t]], V e c t)$. Using Proposition [2.5 from it we obtain the functor $\left.\widetilde{i}_{\mathbb{H}}^{\mathbf{G}}[t]\right]$ : $\operatorname{Rep}(\mathbb{H}, \mathbb{V} e c t) \rightarrow \operatorname{Rep}(\mathbf{G}[[t]], \mathbb{V} e c t)$, which is the right adjoint to $r_{\mathbb{H}}^{\mathbf{G}[[t]]}: \operatorname{Rep}(\mathbf{G}[[t]], \mathbb{V} e c t) \rightarrow$ $\operatorname{Rep}(\mathbb{H}, \mathbb{V} e c t)$; and hence also the functor $\widetilde{i}_{\mathbb{H}}^{\mathbb{G}}: \operatorname{Rep}(\mathbb{H}, \mathbb{V} e c t) \rightarrow \operatorname{Rep}(\mathbb{G})$ with the desired adjointness property.

3.6. Consider the functor $r_{\mathbf{G}}^{\mathbb{G}}: \operatorname{Rep}(\mathbb{G}) \rightarrow \operatorname{Rep}(\mathbf{G}, \mathbb{V} e c t)$ equal to the composition of $r_{\mathbf{G}[[t]]}^{\mathbb{G}}$ : $\operatorname{Rep}(\mathbb{G}) \rightarrow \operatorname{Rep}(\mathbf{G}[[t]], \mathbb{V} e c t)$ and the functor $\mathbb{V} \mapsto \mathbb{V}_{\mathbf{G}^{1}}: \operatorname{Rep}(\mathbf{G}[[t]], \mathbb{V} e c t) \rightarrow \operatorname{Rep}(\mathbf{G}, \mathbb{V} e c t)$ Note that by Lemma [2.7] $r_{\mathbf{G}}^{\mathbb{G}}$ is exact. Its right adjoint, which we will denote by $i_{\mathbf{G}}^{\mathbb{G}}$ is the composition of the "obvious" functor $\operatorname{Rep}(\mathbf{G}, \mathbb{V} e c t) \rightarrow \operatorname{Rep}(\mathbf{G}[[t]], \mathbb{V e c t})$ coming from the homomorphism $\mathbf{G}[[t]] \rightarrow \mathbf{G}$ and the functor $\left.i i_{\mathbf{G}}^{\mathbb{G}}[t]\right]$ studied above. 
More generally, let $P \subset G$ be a parabolic, with the Levi quotient $M$, and let $I_{P} \subset \mathbf{G}[[t]]$ be the corresponding parahoric subgroup. (For $P=B$ we will denote $I_{B}$ simply by $I$, and $M$ by $T)$. Let $\mathbf{I}_{P}$ (resp., $\mathbf{P}, \mathbf{M}$ ) be the corresponding group-objects of Pro(Set) (resp., $\mathbf{S} e t$ ).

In a similar fashion we obtain a pair of mutually adjoint functors $r_{\mathbf{M}}^{\mathbb{G}}: \operatorname{Rep}(\mathbb{G}) \rightarrow$ $\operatorname{Rep}(\mathbf{M}, \mathbb{V} e c t)$ and $i_{\mathbf{M}}^{\mathbb{G}}: \operatorname{Rep}(\mathbf{M}, \mathbb{V} e c t) \rightarrow \operatorname{Rep}(\mathbb{G})$.

Let now $\widehat{G}$ be a central extension of $G((t))$ by means of $G_{m}$ (cf. Sect. 2.14), and let $\operatorname{Rep}_{c}(\widehat{\mathbb{G}})$ be the corresponding category of representations. Since we are given a splitting of $\widehat{\mathbb{G}}$ over $\mathbf{G}[[t]]$, and hence, over $\mathbb{H}$, we have an obvious restriction functor $r_{\mathbb{H}}^{\widehat{\mathbb{G}}}: \operatorname{Rep}_{c}(\widehat{\mathbb{G}}) \rightarrow \operatorname{Rep}(\mathbb{H}, \mathbb{V} e c t)$.

By repeating the construction of the previous subsections, we obtain the functors $i_{\mathbb{H}}^{\widehat{\mathbb{G}}}$ : $\operatorname{Rep}(\mathbb{H}, \mathbb{V} e c t) \rightarrow \operatorname{Rep}_{c}(\widehat{\mathbb{G}})$, and $\widetilde{i}_{\mathbb{H}}^{\mathbb{G}}: \operatorname{Rep}(\mathbb{H}, \mathbb{V} e c t) \rightarrow \operatorname{Rep}_{c}(\widehat{\mathbb{G}})$, such that $\widetilde{i_{\mathbb{H}}}$ is the right adjoint of $r_{\mathbb{H}}^{\widehat{\mathbb{G}}}$, and $i_{\mathbb{H}}^{\widehat{\mathbb{G}}} \simeq \widetilde{i_{\mathbb{H}}^{\mathbb{G}}}$ when $\mathbb{H}$ contains $\mathbf{I}$.

We will denote by $r_{\mathbf{G}}^{\widehat{G}}, i_{\mathbf{G}}^{\widehat{G}}\left(\right.$ resp., $\left.r_{\mathbf{T}}^{\widehat{\mathbb{G}}}, i_{\mathbf{T}}^{\widehat{\mathbb{G}}}\right)$ the corresponding functors between $\operatorname{Rep}_{c}(\widehat{\mathbb{G}})$ and $\operatorname{Rep}(\mathbf{G}, \mathbb{V} e c t)($ resp., $\operatorname{Rep}(\mathbf{T}, \mathbb{V} e c t))$.

3.7. Next we will establish an analogue of Bernstein's geometric lemma, which describes the composition of the functors $i_{\mathbf{T}}^{\widehat{\mathbb{G}}}$ and $r_{\mathbf{T}}^{\widehat{\mathbb{G}}}$, cf. [3].

Let $\Lambda$ be the lattice of co-weights of the maximal torus $T$ of $G$, and $W$-the Weyl group. When we restrict $\widehat{G}$ to $T((t)) \subset G((t))$, the commutator defines a map

$$
T((t)) / T[[t]] \times T[[t]] \rightarrow G_{m}
$$

which factors through $T((t)) / T[[t]] \rightarrow \Lambda$ and $T[[t]] \rightarrow T$. In other words, we have a map

$$
\Lambda \times T \rightarrow G_{m},
$$

which defines a pairing $Q: \Lambda \otimes \Lambda \rightarrow \mathbb{Z}$. This pairing is $W$-invariant, since the central extension of $T((t))$ was induced from that of $G((t))$. For $\lambda \in \Lambda$ we will denote by $\phi_{c Q}(\lambda)$ the character $\mathbf{T} \rightarrow \mathbb{C}^{*}$ equal to

$$
\mathbf{T} \stackrel{Q(\lambda, \cdot)}{\longrightarrow} \mathbf{G}_{m} \stackrel{c}{\rightarrow} \mathbb{C}^{*}
$$

Recall now the affine flag scheme of $G$, which is a strict ind-scheme, equal by definition to $G((t)) / I$ (where $I$ is the Iwahori subgroup), and denoted $\mathrm{Fl}_{G}$. Its existence, i.e., the indrepresentability of the corresponding functor, follows easily from the case of $\mathrm{Gr}_{G}$, cf. [5] .

Recall also that the set of $I$-orbits on $\mathrm{Fl}_{G}$ identifies naturally with $W_{\text {aff }} \simeq \Lambda \ltimes W$-the extended affine Weyl group of $G$. For $w \in W_{a f f}$, let us denote by $\mathrm{Fl}_{G}^{w}$ the corresponding orbit and by $\overline{\mathrm{Fl}}_{G}^{w}$ its closure. Note that $W_{a f f}$ is naturally partially ordered and $\overline{\mathrm{Fl}}_{G}^{w}=\underset{w^{\prime} \leq w}{\cup} \mathrm{Fl}_{G}^{w}$.

Let $\check{T}$ be the Langlands dual torus of $T$ (over $\mathbb{C}$ ), which identifies with the set of unramified characters of T. For $w \in W_{a f f}$ let us denote by $w\left(\rho_{a f f}\right)-\rho_{a f f}$ the character of $T$ equal to the projection on $T$ of the sum of negative affine roots which are turned positive by the action of $w^{-1}$. Let $\mu_{w}$ denote the element in $\check{T}$ equal to the value of $w\left(\rho_{a f f}\right)-\rho_{a f f}: G_{m} \rightarrow \check{T}$ on $q \in \mathbb{C}^{*}$, where $q$ is the order of the residue field of our local field $\mathbf{K}$.

For $w \in W_{a f f}$ and $\Pi \in \operatorname{Rep}(\mathbf{T}, V e c t)$ we define a new representation $w \cdot \Pi$ by setting

$$
w \cdot \Pi:=\Pi^{\bar{w}} \otimes \phi_{c Q}(\lambda) \otimes \mu_{w},
$$

where $w=\lambda \cdot \bar{w}, \lambda \in \Lambda, \bar{w} \in W$, and $\Pi^{w}$ is obtained from $\Pi$ by twisting the $\mathbf{T}$-action using $w$ viewed as an automorphism of $T$.

Proposition 3.8. For a representation $\Pi=(\mathbf{V}, \rho) \in \operatorname{Rep}(\mathbf{T}, V$ ect $)$, the object $r_{\mathbf{T}}^{\widehat{\mathbb{G}}} \circ i_{\mathbf{T}}^{\widehat{\mathbb{G}}}(\Pi)$ can be canonically written as $\underset{w \in W_{a f f}}{\text { lim" }} \mathbf{V}_{w}, \mathbf{V}_{w} \in \operatorname{Rep}(\mathbf{T}, V e c t)$ in such a way that for for $w^{\prime} \leq w$ the 
map $\mathbf{V}_{w} \rightarrow \mathbf{V}_{w^{\prime}}$ is a surjection, and the kernel $\mathbf{V}^{w}:=\operatorname{ker}\left(\mathbf{V}_{w} \rightarrow \underset{w^{\prime}<w}{\oplus} \mathbf{V}_{w^{\prime}}\right)$ is isomorphic to $w \cdot \Pi$.

Proof. Let $\mathbf{F} l_{G}$ be the object of Ind(Set) corresponding to the ind-scheme $\mathrm{Fl}_{G}$. Let us denote by $\mathbf{F} l_{G}^{w}$ and $\overline{\mathbf{F}} l_{G}^{w}$ the corresponding objects of $\mathbf{S} e t$.

Let $\mathbf{I}^{0}$ denote the kernel of the map $\mathbf{I} \rightarrow \mathbf{T}$. Let $\mathbf{S}^{w}$ (resp., $\overline{\mathbf{S}}^{w}$ ) be the preimage of $\mathbf{F} l_{G}^{w}$ (resp., $\overline{\mathbf{F} l}{ }_{G}^{w}$ ) in $\widehat{\mathbb{G}} / \mathbf{I}^{0}$. By construction, $r_{\mathbf{I}}^{\widehat{\mathbb{G}}} \circ i_{\mathbf{T}}^{\widehat{\mathbb{G}}}(\Pi)$ is the inverse limit of

$$
\mathbf{W}_{w}:=\left(\operatorname{Funct}_{c}^{l c}\left(\overline{\mathbf{S}}^{w}, \mathbf{V}\right)\right)_{\mathbf{T} \times \mathbf{G}_{m}} \simeq\left(\operatorname{Funct}^{l c}\left(\overline{\mathbf{S}}^{w}, \mathbf{V}\right)\right)^{\mathbf{T} \times \mathbf{G}_{m}} .
$$

Set $\mathbf{V}_{w}:=\left(\mathbf{W}_{w}\right)_{\mathbf{I}^{0}}$. Since for $w^{\prime} \leq w$, the restriction map $\operatorname{Funct}_{c}^{l c}\left(\overline{\mathbf{S}}^{w}, \mathbf{V}\right) \rightarrow \operatorname{Funct}_{c}^{l c}\left(\overline{\mathbf{S}}^{w^{\prime}}, \mathbf{V}\right)$ is surjective, we obtain that $\mathbf{V}_{w} \rightarrow \mathbf{V}_{w^{\prime}}$ are indeed surjective, by the right-exactness of the functors $(\cdot)_{\mathbf{T}} \times \mathbf{G}_{m}$ and $(\cdot)_{\mathbf{I}^{0}}$.

Using Lemma 2.7 we obtain that

$$
\mathbf{V}^{w}:=\operatorname{ker}\left(\mathbf{V}_{w} \rightarrow \underset{w^{\prime}<w}{\oplus} \mathbf{V}_{w^{\prime}}\right) \simeq\left(\left(\operatorname{Funct}_{c}^{l c}\left(\mathbf{S}^{w}, \mathbf{V}\right)\right)_{\mathbf{T} \times \mathbf{G}_{m}}\right)_{\mathbf{I}^{0}}
$$

Let us choose a splitting $\mathbf{T} \rightarrow \mathbf{B}$, by means of which $\mathbf{T}$ becomes a subgroup of $\mathbf{I}$; let $g \in \mathrm{Fl}_{G}^{w}(\mathbf{K})$ be the $\mathbf{T}$-stable point, and let $S t(g)_{\mathbf{I}}$ be the stabilizer of $g$ in $\mathbf{I}$. We obtain a homomorphism $S t(g)_{\mathbf{I}} \rightarrow \mathbf{T} \times \mathbf{G}_{m}$ and we have:

$$
\left(\left(\operatorname{Funct}_{c}^{l c}\left(\mathbf{S}^{w}, \mathbf{V}\right)\right)_{\mathbf{T} \times \mathbf{G}_{m}}\right)_{\mathbf{I}^{0}} \simeq\left(i_{S t(g)_{\mathbf{I}}}^{\mathbf{I}} \circ r_{\mathbf{T} \times \mathbf{G}_{m}}^{S t()_{\mathbf{I}}}(\Pi)\right)_{\mathbf{I}^{0}} .
$$

Observe that the character of $\mathbf{T}$, corresponding to measures on the homogeneous space $\mathrm{Fl}_{G}^{w}(\mathbf{K}) \simeq \mathbf{I} / S t(g)_{\mathbf{I}}$, equals $\mu_{w}$.

Write $w=\lambda \cdot \bar{w}, \lambda \in \Lambda, \bar{w} \in W$. Observe now that the pull-back of $\Pi$ under the composition

$$
\mathbf{T} \rightarrow S t(g)_{\mathbf{I}} \rightarrow \mathbf{I} \times \mathbf{G}_{m} \rightarrow \mathbf{I} \rightarrow \mathbf{T}
$$

is naturally isomorphic to $\Pi^{\bar{w}}$, and the pull-back of the character $c: \mathbf{G}_{m} \rightarrow \mathbb{C}^{*}$ under $\mathbf{T} \rightarrow$ $S t(g)_{\mathbf{I}} \rightarrow \mathbf{I} \times \mathbf{G}_{m} \rightarrow \mathbf{G}_{m}$ is $\phi_{c Q}(\lambda)$.

This implies that $\mathbf{V}^{w} \simeq w \cdot \Pi$ as $\mathbf{T}$-representations.

3.9. One can formulate an analog of Proposition 3.8 describing the composition of the functors $r_{\mathbf{G}}^{\widehat{\mathbb{G}}} \circ i_{\mathbf{G}}^{\widehat{\mathbb{G}}}: \operatorname{Rep}(\mathbf{G}, \mathbb{V} e c t) \rightarrow \operatorname{Rep}(\mathbf{G}, \mathbb{V} e c t):$

Set $\operatorname{Gr}_{G}:=G((t)) / G[[t]]$, and recall that $G[[t]]$-orbits on $\operatorname{Gr}_{G}$ are in a natural bijection with the partially ordered set $\Lambda^{+}$of dominant weights.

For every $\lambda \in \Lambda^{+}$, let $g \in \operatorname{Gr}_{G}^{\lambda}$ be a $T$-stable point, and let $S t(g)_{G[[t]]}$ be its stabilizer in $G[[t]]$, so that $\operatorname{Gr}_{G}^{\lambda} \simeq G[[t]] / S t(g)_{G[[t]]}$. Note that since $G^{1}$ is normal in $G[[t]]$, the quotient $G^{1} \backslash \mathrm{Gr}_{G}^{\lambda}$ is a $G$-homogeneous space isomorphic to $G / P^{\lambda}$ for a parabolic $P^{\lambda} \subset G$. Let $M^{\lambda}$ be the Levi quotient of $P^{\lambda}$, and let $\mu_{\lambda}$ be the character of $M^{\lambda}$ corresponding to measures on the homogeneous space $I_{P} / S t(g)_{G[[t]]}(\mathbf{K})$. Note also that for $\lambda$ as above, the character $\phi_{c Q}(\lambda)$ of $\mathbf{T}$ is in fact well-defined as a character of $\mathbf{M}^{\lambda}$.

Recall from the theory of $\mathfrak{p}$-adic groups that for a parabolic $P$ with a Levi quotient $M$ we have a pair of mutually adjoint functors $r_{\mathbf{M}}^{\mathbf{G}}: \operatorname{Rep}(\mathbf{G}, V e c t) \rightarrow \operatorname{Rep}(\mathbf{M}, V e c t)$ and $i_{\mathbf{M}}^{\mathbf{G}}:$ $\operatorname{Rep}(\mathbf{M}, V e c t) \rightarrow \operatorname{Rep}(\mathbf{G}, V e c t)$. 
Proposition 3.10. For a representation $\Pi=(\mathbf{V}, \rho) \in \operatorname{Rep}(\mathbf{G}$, Vect $)$, the object $r_{\mathbf{G}}^{\widehat{\widehat{G}}} \circ i_{\mathbf{G}}^{\widehat{\mathbb{G}}}(\Pi)$ can be canonically written as "lim" $\mathbf{V}_{\lambda}, \mathbf{V}_{\lambda} \in \operatorname{Rep}(\mathbf{G}$, Vect $)$ in such a way that for $\lambda^{\prime} \leq \lambda$ the map $\mathbf{V}_{\lambda} \rightarrow \mathbf{V}_{\lambda^{\prime}}$ is a surjection, and the kernel $\mathbf{V}^{\lambda}:=\operatorname{ker}\left(\mathbf{V}_{\lambda} \rightarrow \underset{\lambda^{\prime}<\lambda}{\oplus} \mathbf{V}_{\lambda^{\prime}}\right)$ is canonically isomorphic to $i_{\mathbf{M}^{\lambda}}^{\mathbf{G}}\left(r_{\mathbf{M}^{\lambda}}^{\mathbf{G}}(\Pi) \otimes \mu_{\lambda} \otimes \phi_{c Q}(\lambda)\right)$.

The proof of this proposition is parallel to that of Proposition 3.8

\section{EXAMPLES}

4.1. Assume now that the group $G$ is split, simple and simply-connected. In this case, a data of an extension $\widehat{G}$ is equivalent to that of a $W$-invariant even symmetric bilinear form $Q: \Lambda \otimes \Lambda \rightarrow \mathbb{Z}$ (cf. Sect. 4 of $[4]$ ), and we fix this form to be the minimal one, i.e., $\frac{1}{2 \grave{h}} Q_{0}$, where $Q_{0}$ corresponds to the Killing form, and $\check{h}$ is the dual Coxeter number.

We have previously worked with a fixed character $\mathbf{G}_{m} \rightarrow \mathbb{C}^{*}$, but now we will consider all representations of the group $\widehat{\mathbb{G}}$. For a thick subgroup $\mathbb{H} \subset \mathbf{G}[[t]]$ we have the corresponding functors $i_{\mathbb{H} \times \mathbf{G}_{m}}^{\widehat{\mathbb{G}}}, r_{\mathbb{H}}^{\widehat{\widehat{G}}} \times \mathbf{G}_{m}$ between $\operatorname{Rep}(\mathbb{G})$ and $\operatorname{Rep}\left(\mathbb{H} \times \mathbf{G}_{m}, \mathbb{V} e c t\right)$.

Let $\Lambda_{\text {aff }}$ be the lattice $\Lambda \oplus \mathbb{Z}$; which identifies with the quotient of $\mathbf{T} \times \mathbf{G}_{m}$ by its maximal compact subgroup, and let $\mathbb{C}\left[\Lambda_{a f f}\right]$ be its group-algebra, viewed as a representation of $\mathbf{T} \times \mathbf{G}_{m}$.

Consider the object $\mathbb{V}:=i_{\mathbf{T} \times \mathbf{G}_{m}}^{\widehat{\widehat{\mathbb{A}}}}\left(\mathbb{C}\left[\Lambda_{a f f}\right]\right) \in \operatorname{Rep}(\widehat{\mathbb{G}})$, studied by Kapranov in $\left[\underline{8}\right.$. Let $\ddot{\mathrm{H}}_{q}$ be the modified Cherednik algebra of loc.cit. 2.3.3. In [8] it was shown that $\ddot{\mathrm{H}}_{q}$ injects into $\operatorname{End}_{\operatorname{Rep}(\widehat{\mathbb{G}})}\left(\mathbb{C}\left[\Lambda_{a f f}\right]\right)$.

By combining the results of 8 and Proposition 3.5 we will establish the following result:

Proposition 4.2. The map $\ddot{\mathrm{H}}_{q} \rightarrow \operatorname{End}_{\operatorname{Rep}(\widehat{\mathbb{G}})}(\mathbb{V})$ is an isomorphism.

Proof. Let $\mathbb{V}^{\text {rat }}$ be the object of $\operatorname{Rep}(\widehat{\mathbb{G}})$ equal to $i_{\mathbf{T}}^{\widehat{\mathbb{G}}} \times \mathbf{G}_{m}\left(\mathbb{C}\left(\check{T} \times G_{m}\right)\right)$, where $\mathbb{C}\left(\check{T} \times G_{m}\right)$ is the field of rational functions on the torus $\check{T} \times G_{m}$, viewed as a $\mathbf{T} \times \mathbf{G}_{m}$-representation. Note that by construction, both $\mathbb{V}$ and $\mathbb{V}^{\text {rat }}$ carry an action of the algebra $\mathbb{C}\left[\Lambda_{a f f}\right]$ by endomorphisms.

Using Proposition 3.8 and Proposition 3.5 we obtain that

$$
\operatorname{Hom}_{\operatorname{Rep}(\widehat{\mathbb{G}})}\left(\mathbb{V}, \mathbb{V}^{r a t}\right) \simeq \underset{w \in \lim _{\overrightarrow{W_{a f f}}}}{\operatorname{Hom}} \operatorname{Hom}_{\Lambda_{a f f}-\bmod }\left(\mathbf{V}_{w}, \mathbb{C}\left(\check{T} \times G_{m}\right)\right)
$$

where $\mathbf{V}^{w}:=\operatorname{ker}\left(\mathbf{V}_{w} \rightarrow \underset{w^{\prime}<w}{\oplus} \mathbf{V}_{w^{\prime}}\right)$ is isomorphic to $w \cdot \mathbb{C}\left[\Lambda_{a f f}\right]$. In particular, we see that the restriction map $\operatorname{Hom}_{\operatorname{Rep}(\widehat{\mathbb{G}})}\left(\mathbb{V}^{r a t}, \mathbb{V}^{r a t}\right) \rightarrow \operatorname{Hom}_{\operatorname{Rep}(\widehat{\mathbb{G}})}\left(\mathbb{V}, \mathbb{V}^{r a t}\right)$ is an isomorphism.

The subquotients $\operatorname{Hom}_{\Lambda_{a f f}-\bmod }\left(\mathbf{V}^{w}, \mathbb{C}\left(\check{T} \times G_{m}\right)\right)$ are all identified with $\mathbb{C}\left(\check{T} \times G_{m}\right)$ as left $\Lambda_{a f f}$-modules, with the right $\Lambda_{a f f}$-module structure twisted by $w$. (see Proposition 3.8). Hence, we obtain a canonical direct sum decomposition

$$
\operatorname{End}_{\operatorname{Rep}(\widehat{\mathbb{G}})}\left(\mathbb{V}^{r a t}\right) \simeq \operatorname{Hom}_{\operatorname{Rep}(\widehat{\mathbb{G}})}\left(\mathbb{V}, \mathbb{V}^{r a t}\right) \simeq \mathbb{C}\left(\check{T} \times G_{m}\right) \ltimes W_{a f f}
$$

Therefore, using the main Theorem 3.3.8 of [8], it suffices to check that the isomorphism (4) coincides with the map

$$
\ddot{\mathrm{H}}_{q}{ }^{r a t} \simeq \mathbb{C}\left(\check{T} \times G_{m}\right) \ltimes W_{a f f} \rightarrow \operatorname{Hom}_{\operatorname{Rep}(\widehat{\mathbb{G}})}\left(\mathbb{V}, \mathbb{V}^{r a t}\right) \rightarrow \operatorname{End}_{\operatorname{Rep}(\widehat{\mathbb{G}})}\left(\mathbb{V}^{r a t}\right)
$$

of [8], Equation 3.3.7. 
Since both isomorphisms preserve the ring structure, it suffices to check that the generators of $\mathbb{C}\left(\check{T} \times G_{m}\right) \ltimes W_{\text {aff }}$ over $\mathbb{C}\left(\check{T} \times G_{m}\right)$, corresponding to the simple reflections under the two homomorphisms, act on $\mathbb{V}^{\text {rat }}$ in the same way.

If $s$ is a simple reflection in $W_{a f f}$, there exists a parahoric $I_{s} \subset G((t))$ such that the corresponding Levi quotient $M_{s}$ is a reductive group of semi-simple rank 1. As in Sect. 3.6 we have an induction functor $i_{\mathbf{M}_{s}}^{\widehat{\mathbb{G}}}: \operatorname{Rep}\left(\mathbf{M}_{s}, V e c t\right) \rightarrow \operatorname{Rep}(\mathbb{G})$, and $\mathbb{V}^{r a t} \simeq i_{\mathbf{M}_{s}}^{\widehat{\mathbb{G}}_{s}} \circ i_{\mathbf{T} \times \mathbf{G}_{m}}^{\mathbf{M}_{s}}\left(\mathbb{C}\left(\check{T} \times G_{m}\right)\right)$, so that the endomorphism of $\mathbb{V}^{r a t}$ corresponding to $s$ via both (4) and the integral operator $\tau_{s}$ of [8] come from the corresponding endomorphisms of $i_{\mathbf{T} \times \mathbf{G}_{m}}^{\mathbf{M}_{s}}\left(\mathbb{C}\left(\check{T} \times G_{m}\right)\right)$.

Therefore, we have reduced the question about the equality of two endomorphisms of $\mathbb{V}^{\text {rat }}$ to a similar question about endomorphisms of $i_{\mathbf{T} \times \mathbf{G}_{m}}^{\mathbf{M}_{s}}\left(\mathbb{C}\left(\check{T} \times G_{m}\right)\right)$ in the theory of p-adic groups. This reduces to the following (well-known) calculation:

Let $G$ be a split reductive group of semi-simple rank 1, and consider the $G(\mathbf{K})$-representation $\mathbf{V}:=i_{\mathbf{T}}^{\mathbf{G}}(\mathbb{C}[\Lambda])$, which identifies with the space of locally-constant compactly supported functions on the quotient $G(\mathbf{K}) / N(\mathbf{K})$, where $N$ is the maximal unipotent subgroup of $G$. We can view $\mathbf{V}$ as a $\check{T}$-family of principal series representations, denoted $\mathbf{V}_{t}, t \in \check{T}$. Let $\mathbf{V}^{\text {rat }}$ be the G-representation $i_{\mathbf{T}}^{\mathbf{G}}(\mathbb{C}(\check{T}))$. As above, we have

$$
\mathbb{C}(\check{T}) \ltimes W \simeq \operatorname{End}_{G(\mathbf{K})}\left(\mathbf{V}^{r a t}\right) \simeq \operatorname{Hom}_{G(\mathbf{K})}\left(\mathbf{V}, \mathbf{V}^{r a t}\right) .
$$

Consider the element $\tau_{s}$ of $\operatorname{Hom}_{\mathbf{G}}\left(\mathbf{V}, \mathbf{V}^{\text {rat }}\right)$ corresponding to the (unique) simple reflection in $W \subset \mathbb{C}(\check{T}) \ltimes W$. Then $\tau_{s}$ gives rise to a map $\mathbf{V}_{t} \rightarrow \mathbf{V}_{s \cdot t}$ defined for $t$ belonging to an open subset of $\check{T}$, and the claim is that this map is given by the meromorphic integral operator $f \mapsto f^{\tau_{s}}$ with

$$
f^{\tau_{s}}(g)=\int_{n \in N(\mathbf{K})} f(g \cdot n \cdot s) .
$$

4.3. Let us go back to the situation, when the parameter $c$ is fixed and unramified. Let $\mathbb{V}_{c}:=i_{\mathbf{T}}^{\widehat{\mathbb{G}}}(\mathbb{C}[\Lambda]) \in \operatorname{Rep}_{c}(\widehat{\mathbb{G}})$ and let $\ddot{H}_{q, c}$ be the specialization of $\ddot{H}_{q}$ at $c$.

Corollary 4.4. We have an isomorphism $\ddot{\mathrm{H}}_{q, c} \simeq \operatorname{End}\left(\mathbb{V}_{c}\right)$.

Proof. By applying Proposition [3.5 and Proposition [3.8 to $\mathbb{V}$ and $\mathbb{V}_{c}$, we obtain that $\operatorname{Hom}_{\operatorname{Rep}(\widehat{\mathbb{G}})}(\mathbb{V}, \mathbb{V})$ is a flat module over $\mathbb{C}\left[\Lambda_{a f f}\right]$, and hence over $\mathbb{C}[\mathbb{Z}]$, whose fiber at $c \in \operatorname{Spec}(\mathbb{C}[\mathbb{Z}])$ identifies with $\operatorname{Hom}_{\operatorname{Rep}_{c}(\mathbb{G})}\left(\mathbb{V}_{c}, \mathbb{V}_{c}\right)$.

In other words, $\operatorname{Hom}_{\operatorname{Rep}_{c}(\mathbb{G})}\left(\mathbb{V}_{c}, \mathbb{V}_{c}\right)$ is isomorphic to the fiber of $\ddot{H}_{q}$ at $c$, which is the same as $\ddot{H}_{q, c}$.

4.5. The representation $\mathbb{V}_{c}$ studied above is an analogue of principal series representations. We will now introduce an object of $\operatorname{Rep}(\mathbb{G})$ which should be thought of as a cuspidal representation of $\mathbb{G}$, although at the moment we do not have a definition of cuspidality.

Let $\Pi$ be an irreducible cuspidal representation of $\mathbf{G}$; and consider $i_{\mathbf{G}}^{\widehat{\mathbb{G}}}(\Pi) \in \operatorname{Rep}_{c}(\widehat{\mathbb{G}})$.

Lemma 4.6. $\operatorname{End}_{\operatorname{Rep}_{c}(\widehat{\mathbb{G}})}\left(i_{\mathbf{G}}^{\widehat{\mathbb{G}}}(\Pi)\right) \simeq \mathbb{C}$.

Proof. Using Proposition 3.5 we have $\operatorname{End}_{\operatorname{Rep}_{c}(\widehat{\mathbb{G}})}\left(i_{\mathbf{G}}^{\widehat{\mathbb{G}}}(\Pi)\right) \simeq \operatorname{Hom}_{\operatorname{Rep}(\mathbf{G}, \mathbb{V e c t})}\left(r_{\mathbf{G}}^{\widehat{\mathbb{G}}} \circ i_{\mathbf{G}}^{\widehat{\mathbb{G}}}(\Pi), \Pi\right)$. 
We claim that the natural map $r_{\mathbf{G}}^{\widehat{\mathbb{G}}} \circ i_{\mathbf{G}}^{\widehat{\mathbb{G}}}(\Pi) \rightarrow \Pi$ is an isomorphism, which would imply the assertion of the lemma. In fact, we claim that all the subquotients $\mathbf{V}^{\lambda}$ of Proposition [3.10 vanish except for $\lambda=0$.

Indeed, by Proposition [3.10 each such subquotient involves the functor $r_{\mathbf{M}^{\lambda}}^{\mathbf{G}}$ applied to $\Pi$, which vanishes, since $\Pi$ was assumed to be cuspidal.

Conjecture 4.7. The objects $i_{\mathrm{G}}^{\widehat{\widehat{G}}}(\Pi)$, for $\Pi$ being a cuspidal representation of $\mathbf{G}$, are irreducible.

4.8. Recall that an object $\Pi \in \operatorname{Rep}(\mathbf{G}, V e c t)$ is called admissible if for every open compact subgroup $\mathbf{H} \subset \mathbf{G}$, the vector space $\Pi^{\mathbf{H}} \simeq \Pi_{\mathbf{H}}$ is finite-dimensional, i.e., belongs to $V$ ect $t_{0}$.

We can give an analogous definition in the case of $\operatorname{Rep}_{c}(\widehat{\mathbb{G}})$ :

Definition 4.9. An object $\Pi \in \operatorname{Rep}_{c}(\widehat{\mathbb{G}})$ is called admissible if for every thick subgroup $\mathbb{H} \subset$ $\mathbf{G}[[t]]$ the object $(\Pi)_{\mathbb{H}} \in \mathbb{V}$ ect belongs, in fact, to Vect.

It is easy to see that the principal series representations $\mathbb{V}_{c}$ are not admissible. However, we have the following assertion:

Proposition 4.10. The representation $i_{\mathbf{G}}^{\widehat{\mathbb{G}}}(\Pi)$, for $\Pi$ being a cuspidal representation of $\mathbf{G}$, is admissible.

Proof. First, we can replace $\mathbb{H}$ by a congruence subgroup $\mathbf{G}^{i}$ : indeed, if $\mathbb{H} \supset \mathbf{G}^{i}$, then the statement for $\mathbf{G}^{i}$ would imply that for $\mathbb{H}$.

Let $\mathbf{G} r_{G}$ (resp., $\mathbf{G} r_{G}^{\lambda}, \overline{\mathbf{G} r}{ }_{G}^{\lambda}$ ) be the objects of $\operatorname{Ind}(\mathbf{S} e t)$ and $\mathbf{S e t}$ corresponding to $\mathrm{Gr}_{G}, \mathrm{Gr}_{G}^{\lambda}$ and $\overline{\mathrm{Gr}}_{G}^{\lambda}$, respectively.

Let $\mathbf{S}^{\lambda}$ (resp., $\overline{\mathbf{S}}^{\lambda}$ ) be the preimage of $\mathbf{G} r_{G}^{\lambda}$ (resp., $\overline{\mathbf{G} r}{ }_{G}^{\lambda}$ ) in $\widehat{\mathbb{G}} / \mathbf{G}^{1}$. As in Proposition 3.8 the object $r_{\mathbf{G}}^{\widehat{G}}[[t]] \circ i_{\mathbf{G}}^{\widehat{G}}(\Pi) \in \operatorname{Rep}(\mathbf{G}[[t]], \mathbb{V} e c t)$ for $\Pi=(\mathbf{V}, \rho) \in \operatorname{Rep}(\mathbf{G}, V e c t)$ is the inverse limit over $\lambda \in \Lambda^{+}$of Funct $_{c}^{l c}\left(\overline{\mathbf{S}}^{\lambda}, \mathbf{V}\right)_{\mathbf{G} \times \mathbf{G}_{m}}$.

Set $\mathbf{W}^{\lambda}:=$ Funct ${ }_{c}^{l c}\left(\mathbf{S}^{\lambda}, \mathbf{V}\right)_{\mathbf{G} \times \mathbf{G}_{m}}$, and we have to show that $\left(\mathbf{W}^{\lambda}\right)_{\mathbf{G}^{i}} \simeq 0$ for all but finitely many $\lambda$ 's.

Let $g \in \operatorname{Gr}_{G}^{\lambda}(\mathbf{K})$ be a $\mathbf{T}$-stable point and $\mathbf{S} t(g)_{G[[t]]}$ its stabilizer in $\mathbf{G}[[t]]$. By definition, we have a homomorphism $\mathbf{S} t(g)_{G[[t]]} \rightarrow \mathbf{G}[[t]] \times \mathbf{G}_{m}$ and

$$
\mathbf{W}^{\lambda} \simeq i_{\mathbf{S} t(g)_{G[[t]]}}^{\mathbf{G}[t]]} \circ r_{\mathbf{G} \times \mathbf{G}_{m}}^{\mathbf{S} t(g)_{G[t]]}}(\Pi) .
$$

Therefore, as representations of $\mathbf{G}[[t]] / \mathbf{G}^{i}$,

$$
\left(\mathbf{W}^{\lambda}\right)_{\mathbf{G}^{i}} \simeq i_{\mathbf{S} t(g)_{G[[t]]}}^{\mathbf{G}\left[[t] / \mathbf{G}^{i} t(g)_{G[[t]]} \cap \mathbf{G}^{i}\right.}\left(\left(r_{\mathbf{G} \times \mathbf{G}_{m}}^{\mathbf{S} t(g)_{G[t]]}}(\Pi)\right)_{\mathbf{S} t(g)_{G[[t]]} \cap \mathbf{G}^{i}} \otimes \mu\right),
$$

where $\mu$ is a character.

Note that as an object of Vect, $\left(r_{\mathbf{G} \times \mathbf{G}_{m}}^{\mathbf{S} t(g)_{G[t]]}}(\Pi)\right)_{\mathbf{S} t(g)_{G[[t]]} \cap \mathbf{G}^{i}}$ is isomorphic to $(\Pi)_{\mathbf{H}^{i}}$, where $\mathbf{H}^{i}$ is the image of $\mathbf{S} t(g)_{G[[t]]} \cap \mathbf{G}^{i}$ under the homomorphism $\mathbf{S} t(g)_{G[[t]]} \rightarrow \mathbf{G}[[t]] \rightarrow \mathbf{G}$. Therefore, the assertion of the proposition follows from the fact that for all but finitely many $\lambda$ 's, the subgroup $\mathbf{H}^{i} \subset \mathbf{G}$ contains the unipotent radical of a non-trivial parabolic. 


\section{The Schwartz SPACE ON $\mathbb{G}$}

5.1. Suppose now that $\mathbf{S}$ is an object of $\mathbf{S} e t$, corresponding to a smooth scheme of finite type $S$ over $\mathbf{K}$. Then it makes sense to consider the space of locally constant compactly supported measures on $S(\mathbf{K})$, denoted $M(\mathbf{S})$. To define it, we choose locally a top degree nowhere vanishing differential form $\omega$ on $S$, which defines a measure $\mu(\omega)$ on $S(\mathbf{K})$. We say that a measure is locally constant if it can be obtained from $\mu(\omega)$ by multiplication by a locally constant function. One readily checks that this definition is independent of the choice of $\omega$.

Suppose now that $\phi: S_{1} \rightarrow S_{2}$ is a smooth map between smooth schemes. In this case, the operation of push-forward of constantly supported measures preserves the subspaces of locally constant ones, i.e., it defines a map $\phi_{!}: M\left(\mathbf{S}_{1}\right) \rightarrow M\left(\mathbf{S}_{2}\right)$.

In particular, if an algebraic group $G$ acts on $S$, we obtain that $M(\mathbf{S})$ is naturally on object of $\operatorname{Rep}(\mathbf{G}, V e c t)$.

For $S$ as above, consider now the object $\mathbb{S} \in \mathbb{S e t}$. It appears that there is no invariant way to assign to $\mathbb{S}$ an object of $\mathbb{V} e c t$, which would be a replacement of locally constant compactly supported measures, and this is similar to the absence of a notion of D-module on $S((t))$, cf. 1 .

In this section we will study this phenomenon first when $S$ is the affine space $A^{n}$, and then when $S$ is an affine algebraic group $G$.

5.2. For any scheme $S$ which is isomorphic to a projective limit of smooth schemes of finite type $S_{i}$ with smooth transition maps $S_{i} \rightarrow S_{j}$, we have $\mathbf{S} \in \operatorname{Pro}(\mathbf{S} e t)$, and we define $M(\mathbf{S}) \in \mathbb{V} e c t$ as $M(\mathbf{S}):=" l i m " M\left(\mathbf{S}_{i}\right)$, where the maps $M\left(\mathbf{S}_{j}\right) \rightarrow M\left(\mathbf{S}_{i}\right)$ for $j \geq i$ are the push-forwards of measures.

Recall that a lattice $L \subset \mathbf{K}((t))^{n}$ is a finitely generated $\mathbf{K}[[t]]$-submodule, which contains $t^{i} \cdot \mathbf{K}[[t]]$ for some $i$. The "standard" lattice is by definition $L_{0}=\mathbf{K}[[t]]$. By abuse of notation, we will denote by the same character $L$ the group-subscheme of $A^{n}((t))$ corresponding to a lattice $L$, and by $\mathbf{L}$ the corresponding object of $\operatorname{Pro}(\mathbf{S} e t)$. Since $L=\underset{{ }_{i}}{\lim }\left(L / t^{i} \cdot L\right)$, we have a well-defined object $M(\mathbf{L}) \in \mathbb{V} e c t$.

5.3. For a finite-dimensional vector space $H$ over $\mathbf{K}$ let $\operatorname{det}(H)=\Lambda^{\text {top }}(H)$ denote its determant line. Let $\mathbf{H}$ and $\operatorname{det}(\mathbf{H})$ be the corresponding objects of $\mathbf{S e t}$, and let $\mu(\operatorname{det}(\mathbf{H}))$ denote the 1dimensional $\mathbb{C}$-vector space of Haar measures on $\operatorname{det}(\mathbf{H}) .{ }^{2}$ Of course, an element of $\mu(\operatorname{det}(\mathbf{H}))$ determines also a Haar measure on $\mathbf{H}$.

Recall that for two lattices $L, L^{\prime} \subset \mathbf{K}((t))^{n}$ we can assign their relative determinant line $\operatorname{det}\left(L, L^{\prime}\right)$ so that $\operatorname{det}\left(L, L^{\prime \prime}\right) \simeq \operatorname{det}\left(L, L^{\prime}\right) \otimes \operatorname{det}\left(L^{\prime}, L^{\prime \prime}\right)$ and for $L \subset L^{\prime}, \operatorname{det}\left(L, L^{\prime}\right)=\operatorname{det}\left(L^{\prime} / L\right)$, where the vector space $L^{\prime} / L$ is, by definition, finite-dimensional. Let $\mu\left(\operatorname{det}\left(\mathbf{L}, \mathbf{L}^{\prime}\right)\right)$ be the corresponding line of Haar measures.

Lemma 5.4. For $L \subset L^{\prime}$ we have a canonical morphism $M\left(\mathbf{L}^{\prime}\right) \rightarrow M(\mathbf{L}) \otimes \mu\left(\operatorname{det}\left(L, L^{\prime}\right)\right)$.

Proof. Let $L^{\prime \prime}$ be a sublattice in $L$. By definition, for every such $L^{\prime \prime}$ we must construct a morphism $M\left(\mathbf{L}^{\prime} / \mathbf{L}^{\prime \prime}\right) \rightarrow M\left(\mathbf{L} / \mathbf{L}^{\prime \prime}\right) \otimes \mu\left(\operatorname{det}\left(\mathbf{L}, \mathbf{L}^{\prime}\right)\right)$. The required map is defined as a composition:

$$
\begin{aligned}
& M\left(\mathbf{L}^{\prime} / \mathbf{L}^{\prime \prime}\right) \simeq \operatorname{Funct}_{c}^{l c}\left(\mathbf{L}^{\prime} / \mathbf{L}^{\prime \prime}\right) \otimes \mu\left(\operatorname{det}\left(\mathbf{L}^{\prime \prime}, \mathbf{L}^{\prime}\right)\right) \rightarrow \operatorname{Funct}_{c}^{l c}\left(\mathbf{L} / \mathbf{L}^{\prime \prime}\right) \otimes \mu\left(\operatorname{det}\left(\mathbf{L}^{\prime \prime}, \mathbf{L}^{\prime}\right)\right) \simeq \\
& \operatorname{Funct}_{c}^{l c}\left(\mathbf{L} / \mathbf{L}^{\prime \prime}\right) \otimes \mu\left(\operatorname{det}\left(\mathbf{L}^{\prime \prime}, \mathbf{L}\right)\right) \otimes \mu\left(\operatorname{det}\left(\mathbf{L}, \mathbf{L}^{\prime}\right)\right) \simeq M\left(\mathbf{L} / \mathbf{L}^{\prime \prime}\right) \otimes \mu\left(\operatorname{det}\left(\mathbf{L}, \mathbf{L}^{\prime}\right)\right),
\end{aligned}
$$

where the arrow corresponds to the ordinary restriction of functions.

\footnotetext{
${ }^{2}$ Properly speaking, $\operatorname{det}(H)$ is a super-vector space; however, in this paper it will appear only via $\mu(\operatorname{det}(H))$, so the difficulties associated with the sign are irrelevant.
} 
Finally, we are ready to define the object $M\left(\mathbb{A}^{n}\right) \in \mathbb{V} e c t$, which we propose as a candidate for the Schwartz space of functions on $A^{n}(\mathbf{F})$ :

$$
M\left(\mathbb{A}^{n}\right):=\underset{L}{\lim } M(\mathbf{L}) \otimes \mu\left(\operatorname{det}\left(\mathbf{L}, \mathbf{L}_{0}\right)\right)
$$

where $\lim _{\text {is }}$ taken in the category $\mathbb{V} e c t$, and the arrows are given by the lemma above.

It is easy to see that the action of $A^{n}((t))$ on itself by translations makes $M\left(\mathbb{A}^{n}\right)$ an object of $\operatorname{Rep}\left(\mathbb{A}^{n}\right)$.

Recall also that the group-indscheme $G L_{n}((t))$, which acts naturally on $A^{n}((t))$, has a canonical central extension $\widehat{G L}_{n}$ by means of $G_{m}$, whose $S$-points for a test-scheme $S$ are pairs $g \in \operatorname{Hom}\left(S, G L_{n}((t))\right)$ and a trivialization of the line bundle $\operatorname{det}\left(g \cdot L_{0}, L_{0}\right)$ on $S$.

Theorem 5.5. The action of $G L_{n}((t))$ on $A^{n}((t))$ makes $M\left(\mathbb{A}^{n}\right)$ an object of $\operatorname{Rep}\left(\widehat{\mathbb{G L}}_{n}\right)$, where $\mathbf{G}_{m} \subset \widehat{\mathbb{G L}}_{n}$ acts via the character $\mathbf{G}_{m} \rightarrow \mathbb{Z} \stackrel{1 \mapsto q}{\longrightarrow} \mathbb{C}^{*}$.

Proof. By construction, as an object of $\mathbb{V e c t}$,

$$
M\left(\mathbb{A}^{n}\right) \simeq " \lim " M\left(\mathbf{L} / \mathbf{L}^{\prime}\right) \otimes \mu\left(\operatorname{det}\left(\mathbf{L}, \mathbf{L}_{0}\right)\right)
$$

where the inverse limit is taken over the partially ordered set of pairs of lattices $L^{\prime} \subset L$ with $\left(L_{1}^{\prime} \subset L_{1}\right) \leq\left(L_{2}^{\prime} \subset L_{2}\right)$ if and only if $L_{1} \subset L_{2}$ and $L_{1}^{\prime} \supset L_{2}^{\prime}$.

For clarity, let us first define an action of the abstract group $\widehat{G L}_{n}(\mathbf{K})$ on $M\left(\mathbb{A}^{n}\right)$. For a pair of lattices $L^{\prime} \subset L$ and $g \in G L_{n}((t))(\mathbf{K})$, the action of $g$ defines an isomorphism $M\left(\mathbf{L} / \mathbf{L}^{\prime}\right) \simeq M(g$. $\left.\mathbf{L} / g \cdot \mathbf{L}^{\prime}\right)$ and an isomorphism $\operatorname{det}\left(L, L_{0}\right) \simeq \operatorname{det}\left(g \cdot L, g \cdot L_{0}\right) \simeq \operatorname{det}\left(g \cdot L, L_{0}\right) \otimes \operatorname{det}\left(L_{0}, g \cdot L_{0}\right)$. Hence, if we lift $g$ to an element of $\widehat{G L}_{n}(\mathbf{K})$, we obtain an isomorphism $\operatorname{det}\left(L, L_{0}\right) \simeq \operatorname{det}\left(g \cdot L, L_{0}\right)$, i.e., we obtain a desired action.

Let us now repeat this construction in order to obtain an action map $\widehat{\mathbb{G L}}_{n} \times M\left(\mathbb{A}^{n}\right) \rightarrow$ $M\left(\mathbb{A}^{n}\right)$. Let us write $\widehat{G L}_{n}$ as "lim" $S_{k}$, and $S_{k}=$ "lim" $S_{k, l}$ with $S_{k, l} \in S c h^{f t}$. Set $\mathbb{S}_{k}$ (resp., $\mathbf{S}_{k, l}$ ) to be the corresponding objects of $\operatorname{Pro}(\mathbf{S} e t)$ (resp., $\left.\mathbf{S} e t\right)$.

Recall that if $S$ is a scheme, there is a notion of an $S$-family of lattices in $\mathbf{K}((t))^{n}$, which is in fact the same as an $S$-point of the affine Grassmannian of $G L_{n}$. If $L$ and $L^{\prime}$ are two $S$-families of lattices with $L^{\prime} \subset L$, then the quotient $L / L^{\prime}$ is a vector bundle on $S$.

For a pair of lattices $L^{\prime} \subset L \subset \mathbf{K}((t))^{n}$ and an index $k$, using the action of $G L_{n}((t))$ on $\mathrm{Gr}_{G L_{n}}$, we obtain the $S_{k}$-families of lattices that we will denote by $S_{k} \cdot L^{\prime} \subset S_{k} \cdot L$. Moreover, there exists another pair of lattices $L_{1}^{\prime} \subset L_{1}$, thought of as constant $S_{k}$-families, such that $L_{1}^{\prime} \subset S_{k} \cdot L^{\prime}$ and $S_{k} \cdot L \subset L_{1}$. Consider the quotients

$$
H_{S_{k}}:=S_{k} \cdot L^{\prime} / L_{1}^{\prime} \subset H_{S_{k}}^{\prime}:=S_{k} \cdot L / L_{1}^{\prime} \subset H_{S_{k}}^{\prime \prime}:=L_{1} / L_{1}^{\prime}
$$

as vector bundles on $S_{k}$. Note that both $H_{S_{k}}^{\prime} / H_{S_{k}}$ and $H_{S_{k}}^{\prime \prime}$ are trivial bundles with fibers $L / L^{\prime}$ and $L_{1} / L_{1}^{\prime}$, respectively. By the definition of $\widehat{G L}_{n}$, the line bundle $\operatorname{det}\left(H_{S_{k}}^{\prime \prime} / H_{S_{k}}^{\prime}\right)$ is identified with the trivial line bundle with fiber $\operatorname{det}\left(L, L_{1}\right)$. Finally, there exists an index $l$, so that $H_{S_{k}}$ and $H_{S_{k}}^{\prime}$, together with their embeddings into $H_{S_{k}}^{\prime \prime}$, come from vector bundles on $S_{k, l}$, which we will denote by $H_{S_{k, l}}$ and $H_{S_{k, l}}^{\prime}$, respectively.

We need to construct an action map $\mathbf{S}_{k, l} \times\left(M\left(\mathbf{L}_{1} / \mathbf{L}_{1}^{\prime}\right) \otimes \mu\left(\operatorname{det}\left(\mathbf{L}_{1}, \mathbf{L}\right)\right)\right) \rightarrow M\left(\mathbf{L} / \mathbf{L}^{\prime}\right)$, which on the level of $\mathbf{K}$-points amounts to the one constructed above. For that, by Zariski localizing 
$S_{l, k}$, we may assume that the vector bundle $H_{S_{k, l}}^{\prime}$ on $S_{k, l}$ can be trivialized, i.e., $H_{S_{k, l}}^{\prime} \simeq$ $H^{\prime} \times S_{k, l}$.

Thus, we have a map $\mathbf{S}_{k, l} \times \mathbf{H}^{\prime} \rightarrow \mathbf{L}_{1} / \mathbf{L}_{1}^{\prime}$, such that the corresponding map $\mathbf{S}_{k, l} \times \mathbf{H}^{\prime} \rightarrow$ $\mathbf{S}_{k, l} \times \mathbf{L}_{1} / \mathbf{L}_{1}^{\prime}$ is proper (cf. Sect. 3.2). Therefore, it defines an action map

$$
\mathbf{S}_{k, l} \times \operatorname{Funct}_{c}^{l c}\left(\mathbf{L}_{1} / \mathbf{L}_{1}^{\prime}\right) \rightarrow \operatorname{Funct}_{c}^{l c}\left(\mathbf{H}^{\prime}\right) .
$$

By tensoring with $\mu\left(\operatorname{det}\left(\mathbf{H}^{\prime}\right)\right)$ we obtain an action map $\mathbf{S}_{k, l} \times\left(M\left(\mathbf{L}_{1} / \mathbf{L}_{1}^{\prime}\right) \otimes \mu\left(\operatorname{det}\left(\mathbf{L}_{1}, \mathbf{L}\right)\right)\right) \rightarrow$ $M\left(\mathbf{H}^{\prime}\right)$.

Similarly, we have a map $\mathbf{S}_{k, l} \times \mathbf{H}^{\prime} \rightarrow \mathbf{L} / \mathbf{L}^{\prime}$, and by integration we obtain an action map $\mathbf{S}_{k, l} \times M\left(\mathbf{H}^{\prime}\right) \rightarrow M\left(\mathbf{L} / \mathbf{L}^{\prime}\right)$. The composition

$$
\begin{aligned}
& \operatorname{Hom}\left(\mathbf{S}_{k, l} \otimes M\left(\mathbf{H}^{\prime}\right), M\left(\mathbf{L} / \mathbf{L}^{\prime}\right)\right) \times \operatorname{Hom}\left(\mathbf{S}_{k, l} \otimes M\left(\mathbf{L}_{1} / \mathbf{L}_{1}^{\prime}\right), M\left(\mathbf{H}^{\prime}\right)\right) \rightarrow \\
& \operatorname{Hom}\left(\left(\mathbf{S}_{k, l} \times \mathbf{S}_{k, l}\right) \otimes M\left(\mathbf{L}_{1} / \mathbf{L}_{1}^{\prime}\right), M\left(\mathbf{L} / \mathbf{L}^{\prime}\right)\right) \rightarrow \operatorname{Hom}\left(\mathbf{S}_{k, l} \otimes M\left(\mathbf{L} / \mathbf{L}^{\prime}\right), M(\mathbf{H})\right)
\end{aligned}
$$

yields the desired action.

5.6. Let now $G$ be an algebraic group over $\mathbf{K}$. Let $G((t))$ be the corresponding loop group and $\widehat{G}$ its central extension $1 \rightarrow G_{m} \rightarrow \widehat{G} \rightarrow G((t)) \rightarrow 1$, as in Sect. 2.14. Let us fix a character $c: \mathbf{G}_{m} \rightarrow \mathbb{C}^{*}$. We will now define an object $M_{c}(\mathbb{G}) \in \mathbb{V e c t}$, which will underly an object $\left(M_{c}(\mathbb{G}), \rho\right) \in \operatorname{Rep}_{c}(\widehat{\mathbb{G}})$.

For every integer $i$ consider the trivial representation $\mathbb{C}$ of the corresponding congruence subgroup $\mathbf{G}^{i}$ and consider $\left.i_{\mathbf{G}^{i}} \widehat{\mathbb{G}}^{i}\right) \otimes \mu\left(\mathbf{G}[[t]] / \mathbf{G}^{i}\right) \in \operatorname{Rep}_{c}(\widehat{\mathbb{G}})$, where $\mu\left(\mathbf{G}[[t]] / \mathbf{G}^{i}\right)$ is the 1dimensional space of left-invariant Haar measures on the group $\left(\mathbf{G}[[t]] / \mathbf{G}^{i}\right)^{\text {top }}$.

By the construction of the functor $i_{\mathbf{G}^{i}}^{\widehat{\mathbb{G}}^{i}}$ via compactly supported functions on $\widehat{\mathbb{G}} / \mathbf{G}^{i}$, for $j \geq i$ we have the morphisms

$$
i_{\mathbf{G}^{j}}^{\widehat{\mathbb{G}}}(\mathbb{C}) \otimes \mu\left(\mathbf{G}[[t]] / \mathbf{G}^{j}\right) \rightarrow i_{\mathbf{G}^{i}}^{\widehat{\mathbb{G}}^{i}}(\mathbb{C}) \otimes \mu\left(\mathbf{G}[[t]] / \mathbf{G}^{i}\right)
$$

given by fiber-wise integration. Set $M_{c}(\mathbb{G}):=\lim i_{\mathbf{G}^{j}}^{\mathbb{G}}(\mathbb{C})$, where $\lim$ is taken in $\mathbb{V} e c t$.

For example, it is easy to see that when $G \simeq A^{n}$ (and the central extension is trivial), the space $M(\mathbb{G})$ obtained in this way identifies canonically with $M\left(\mathbb{A}^{n}\right)$ considered above.

5.7. Since each $\mathbf{G}^{i}$ is a normal subgroup in $\mathbf{G}[[t]]$, the terms of the inverse system defining $M_{c}(\mathbb{G})$ carry a commuting $\mathbf{G}[[t]]$-action on the right, which is respected by the arrows. Hence, $M_{c}(\mathbb{G})$ carries an additional $\mathbf{G}[[t]]$-action "on the right", which commutes with the action of $\widehat{\mathbb{G}}$ "on the left".

This $\widehat{\mathbb{G}}-\mathbf{G}[[t]]$-module structure on $M_{c}(\mathbb{G})$ allows to reinterpret the functor $i_{\mathbb{H}}^{\widehat{\mathbb{G}}}$ introduced earlier:

Recall that the functor of tensor product $V e c t \times V e c t \rightarrow V e c t$ extends naturally to $\mathbb{V e c t}$ :

$$
\left(" \lim " \mathbf{V}_{i}\right) \otimes\left(" \lim _{\longleftarrow} \mathbf{W}_{j}\right):=" \lim "\left(\mathbf{V}_{i} \otimes \mathbf{W}_{j}\right) .
$$

Let $\mathbb{H} \subset \mathbf{G}[[t]]$ be a thick subgroup, and let $\Pi=(\mathbb{V}, \rho)$ be an object of $\operatorname{Rep}(\mathbb{H}, \mathbb{V} e c t)$. Consider the tensor product $M_{c}(\mathbb{G}) \otimes \mathbb{V} \in \mathbb{V}$ ect. The diagonal action of $\mathbb{H}$ makes it into an object of $\operatorname{Rep}(\mathbb{H}, \mathbb{V} e c t)$, which carries a commuting $\widehat{\mathbb{G}}$-action. Hence, $\left(M_{c}(\mathbb{G}) \otimes \mathbb{V}\right)_{\mathbb{H}}$ is naturally an object of $\operatorname{Rep}(\mathbb{G})$.

The following is straightforward from the definitions:

Lemma 5.8. We have a natural isomorphism in $\operatorname{Rep}(\mathbb{G}): i_{\mathbb{H}}^{\widehat{\mathbb{G}}}(\Pi) \simeq\left(M_{c}(\mathbb{G}) \otimes \mathbb{V}\right)_{\mathbb{H}}$. 
5.9. Since we think of $M_{c}(\mathbb{G})$ as the space of functions on the group $\mathbb{G}$, it is natural to expect that the $\mathbf{G}[[t]]$-action on $M_{c}(\mathbb{G})$ considered above extends to an action "on the right" of the entire group $\mathbb{G}=\mathbf{G}((t))$, corresponding to right translations. The existence of such an action is given by the theorem below.

Let $\mathfrak{g}$ be the Lie algebra of $G$, let $G L_{\mathfrak{g}}((t))$ be the corresponding loop group, and let $\widehat{G L}_{\mathfrak{g}}$ be its canonical central extension as in Theorem 5.5 Let $\widehat{G}_{0}$ be the central extension of $G((t))$ induced from $\widehat{G L}_{\mathfrak{g}}$ by means of the adjoint action. For example, if $G$ is simple and simplyconnected, the extension $\widehat{G}_{0}$ corresponds to the pairing $\Lambda \otimes \Lambda \rightarrow \mathbb{Z}$ given by the Killing form.

Let $\widehat{G}^{\prime}$ be the central extension of $G((t))$ equal to the Baer sum of $\widehat{G}_{0}$ and the original extension $\widehat{G}$. Let $c^{\prime}$ be the character of $\mathbf{G}_{m} \subset \widehat{\mathbb{G}}^{\prime}$ equal to the inverse of the product of $c$ and $c_{0}$, where $c_{0}$ is the character $\mathbf{G}_{m} \rightarrow \mathbb{Z} \stackrel{1 \mapsto q}{\longrightarrow} \mathbb{C}^{*}$.

Theorem 5.10. We have a canonical action of $\widehat{\mathbb{G}}^{\prime}$ on $M_{c}(\mathbb{G})$, with $\mathbf{G}_{m} \subset \widehat{\mathbb{G}}^{\prime}$ acting by the character $c^{\prime}$. This action extends the natural action of $\mathbf{G}[[t]]$ on $M_{c}(\mathbb{G})$ "on the right" and commutes with the action of $\widehat{\mathbb{G}}$ "on the left".

5.11. Proof of Theorem [5.10, Let us first construct an action of the abstract group $\widehat{G}^{\prime}(\mathbf{K})$ on $M_{c}(\mathbb{G})$. For an integer $i$ and a point $g \in G((t))(\mathbf{K})$, there exists an integer $j$ such that $g^{-1}\left(\mathbf{G}^{j}\right) g$ is contained in $\mathbf{G}^{i}$; therefore, the right multiplication map $\mathbf{G}((t)) \times g \rightarrow \mathbf{G}((t))$ descends to a well-defined map $\mathbf{G}((t)) / \mathbf{G}^{j} \times g \rightarrow \mathbf{G}((t)) / \mathbf{G}^{i}$.

In particular, if we lift $g$ to an element of $\widehat{G}(\mathbf{K})$, we obtain a map

$$
i_{\mathbf{G}^{j}}^{\widehat{\mathbb{G}}^{j}}(\mathbb{C}) \otimes \mu\left(\mathbf{G}^{i} / g^{-1}\left(\mathbf{G}^{j}\right) g\right) \rightarrow i_{\mathbf{G}^{i}}^{\widehat{\mathbb{G}}^{i}}(\mathbb{C}),
$$

which commutes with the left $\widehat{\mathbb{G}}$-action.

We claim now that a lift of $g$ to an element of $\widehat{G}_{0}(\mathbf{K})$ define an identification of the line $\mu\left(\mathbf{G}^{i} / g^{-1}\left(\mathbf{G}^{j}\right) g\right)$ with $\mu\left(\mathbf{G}^{i} / \mathbf{G}^{j}\right)$. Indeed, let $\mathfrak{g}^{i}$ be the Lie subalgebra in $\mathfrak{g}((t))$ corresponding to the congruence subgroup $\mathbf{G}^{i}$, then

$$
\mu\left(\mathbf{G}^{i} / \mathbf{G}^{j}\right) \simeq \mu\left(\operatorname{det}\left(\mathfrak{g}^{i} / \mathfrak{g}^{j}\right)\right) ; \mu\left(\mathbf{G}^{i} / g^{-1}\left(\mathbf{G}^{j}\right) g\right) \simeq \mu\left(\operatorname{det}\left(\mathfrak{g}^{i} / g^{-1} \cdot \mathfrak{g}^{j}\right)\right),
$$

and

$$
\operatorname{det}\left(\mathfrak{g}^{i} / \mathfrak{g}^{j}\right) \simeq \operatorname{det}\left(\mathfrak{g}^{i} / g^{-1} \cdot \mathfrak{g}^{j}\right) \otimes \operatorname{det}\left(g \cdot \mathfrak{g}^{j}, \mathfrak{g}^{j}\right) \simeq \operatorname{det}\left(\mathfrak{g}^{i} / g^{-1} \cdot \mathfrak{g}^{j}\right) \otimes \operatorname{det}\left(g \cdot \mathfrak{g}^{0}, \mathfrak{g}^{0}\right)
$$

Therefore, if we take the Baer product of the extensions $\widehat{\mathbb{G}}$ and $\widehat{\mathbb{G}}_{0}$ we obtain an action of the group of $\mathbf{K}$-points of $\widehat{\mathbb{G}}^{\prime}$ on $M_{c}(\mathbb{G})$. Therefore, by passing to inverses, we obtain on $M_{c}(\mathbb{G})$ an action of $\widehat{G}^{\prime}(\mathbf{K})$, commuting with the left action of $\widehat{\mathbb{G}}$ and the prescribed value of the central character.

The fact the constructed point-wise action gives rise to a well-defined action map $\widehat{\mathbb{G}}^{\prime} \times$ $M_{c}(\mathbb{G}) \rightarrow M_{c}(\mathbb{G})$ follows by considering families, as in the proof of Theorem [5.5.

Namely, if $\widehat{G} \underset{G((t))}{\times} \widehat{G}_{0}=" \underline{l i m} " S_{k}, S_{k}=" \stackrel{l i m}{\longleftarrow} S_{k, l}$ with $S_{k, l} \in S c h^{f t}$, for every pair of indices $i, k$ there exists a large enough index $j$, such that the group-subscheme $\operatorname{Ad}_{S_{k}}\left(G^{j}\right) \subset G((t)) \times S_{k}$ is contained in $G^{i} \times S_{k}$. Moreover, the relative determinant line $\operatorname{det}\left(\mathfrak{g}^{i} / \operatorname{Ad}_{S_{k}}\left(\mathfrak{g}^{j}\right)\right)$ is identified with the constant line bundle with fiber $\operatorname{det}\left(\mathfrak{g}^{j}, \mathfrak{g}^{i}\right)$.

We have the map

$$
\left(\widehat{G} / G^{j}\right) \times S_{k} \simeq\left(\widehat{G} \times S_{k}\right) / \operatorname{Ad}_{S_{k}}\left(G^{j}\right) \rightarrow \widehat{G} / G^{i},
$$


which comes from a map $\widehat{G} / G^{j} \times S_{k, l} \rightarrow \widehat{G} / G^{i}$ defined for a sufficiently large index $l$. The resulting map $\widehat{G} / G^{j} \times S_{k, l} \rightarrow \widehat{G} / G^{i} \times S_{k, l}$ is smooth over every finite-dimensional subscheme of $\widehat{G} / G^{i}$.

Integration along the fiber defines the desired map

$$
\mathbf{S}_{k, l} \times\left(i_{\mathbf{G}^{j}}^{\widehat{\widehat{C}}}(\mathbb{C}) \otimes \mu\left(\operatorname{det}\left(\mathfrak{g}^{j}, \mathfrak{g}^{i}\right)\right)\right) \rightarrow i_{\mathbf{G}^{i}}^{\widehat{\widehat{C}}}(\mathbb{C}) .
$$

\section{REFERENCES}

[1] S. Arkhipov, D Gaitsgory, Differential operators on the loop group via chiral algebras, IMRN 2002 no. 4, 165-210.

[2] M. Artin, B. Mazur, Etale Homotopy, LNM 100, Springer-Verlag, Berlin-New York (1969).

[3] J. Bernstein, A. Zelevinsky, Induced representations of reductive p-adic groups I, Ann. Sci. Ec. Norm. Sup. 10, (1997), 441-472.

[4] J.-L. Brylinski, P. Deligne, Central extensions of reductive groups by $K_{2}$, Publ. Math. IHES 94 (2001), $5-85$.

[5] D. Gaitsgory, Construction of central elements in the affine Hecke algebra via nearby cycles, Inv. Math. 144 (2001), 253-280.

[6] V. Ginzburg, M. Kapranov, E. Vasserot, Residue construction of Hecke algebras, Adv. Math. 128 (1997), $1-19$.

[7] A. Grothendieck, SGA 4, Exposé I, Lecture Notes in Mathematics 269, Springer-Verlag, Berlin-New York, 1972.

[8] M. Kapranov, Double affine Hecke algebras and 2-dimensional local fields, J. Amer. Math. Soc. 14 (2001), 239-262.

D.K.: Einstein Institute of Mathematics, the Hebrew University of Jerusalem, Givat Ram, Jerusalem, 91904, Israel;

D.G.: Department of Mathematics, The University of Chicago, 5734 University Ave., Chicago, IL, 60637, USA.

E-mail address: kazhdan@math.huji.ac.il; gaitsgde@math.uchicago.edu 